\title{
Neuron glial antigen 2 in the striatum of parkinsonian rats presenting L-DOPA induced dyskinesia
}

\author{
Glauce Nascimento ${ }^{1}$, Mariza Bortolanza $^{1}$, and Elaine Del Bel ${ }^{1}$ \\ ${ }^{1}$ Affiliation not available
}

May 19, 2021

Nascimento G.C.*1, Bortolanza, M.*1, Bribian, A²., Leal-Luiz, G.C. ${ }^{1}$, López-Mascaraque L ${ }^{2 * *}$., Del-Bel, E. ${ }^{1 * *}$

* The authors contributed equally to the work

1 Department of Oral and Basic Biology, Faculty of Odontology of Ribeirao Preto, University of Sao Paulo, 14040-904, Ribeirao Preto, SP, Brazil.

2 Department of Molecular, Cellular, and Developmental Neurobiology, Instituto Cajal -CSIC-, Spanish National Research Council, Avda. Dr. Arce 37, 28002 Madrid, Spain.

Running title: NG2-Glia in the Striatum of Dyskinetic Rats

Funding information: EDB - CAPES/COFECUB Grant/Award Number: 88887.192409/2018-00; CAPES/DAAD Grant/Award Number: 88881.198836/2018-01; FAPESP, Grant/Award Numbers: 2017/243040, 2014/25029-4. GCN - FAPESP, Grant/Award Number: 2015/03053-3 and 2018/05146-7; PNPD CNPQ, 88882.317597/2019-01. LL-M: Research Grants from the MICINN (PID2019-105218RB-I00).

Word Count: 5.060

**Corresponding authors:

Prof. Elaine Del Bel, Department of Basic and Oral Biology, Faculty of Odontology of Ribeirao Preto, University of Sao Paulo (USP), Av do Café s/n; Brazil; Tel: +55 163315 4050, Fax: +55 1636330999 , e-mail: eadelbel@usp.br

Dr. Laura López-Mascaraque Department of Molecular, Cellular, and Developmental Neurobiology, Instituto Cajal -CSIC-, Spanish National Research Council, Avda. Dr. Arce 37, 28002 Madrid, Spain

\section{ABSTRACT (232 words)}

Nerve/glial antigen 2 glial cells (NG2-glia) are a uniformly distributed pool of cells in the central nervous system. In addition, to serve as oligodendrocytes progenitors, NG2-glia might also fulfil physiological roles, although such functions' mechanistic nature remains unclear. Here, we investigated NG2-glia expression in the striatum of 6-hydroxydopamine lesioned Wistar adult male rats presenting L-DOPA induced dyskinesia (LID). Immunoconfocal morphometry, immunohistochemistry, and western blot techniques were performed to label NG2-glia, astrocyte (GFAP), and microglia (OX-42) in the striatum. Our data revealed that the L-DOPA-induced dyskinesia produces a decrease of the NG2-glia density in the striatum, an area critical to Parkinson's disease pathophysiology L-DOPA induced dyskinesia instalment. NG2-glia presented a negative correlation with L-DOPA induced dyskinesia score opposing to GFAP and OX-42 immunopositive cells. The doxycycline antidyskinetic therapy induced a robust increment of NG2-glia restoring standards beforehand L-DOPA induced dyskinesia manifestation. The morphometric analysis identified a NG2 reactive phenotype 
in the striatum of parkinsonian rats, under L-DOPA treatment, not altered by doxycycline. NG2-glia immunolabeled cells do not colocalize with OX-42 or GFAP-labeled cells. In conclusion, our findings provide the first description of the distribution and morphological changes of NG2-glia in the parkinsonian and dyskinetic rats' striatum. The NG2-glia expression rise in the striatum positively correlated to the decrease in L-DOPA induced dyskinesia scores. The results reveal an aspect of the NG2-glia as a potential therapeutic target for Parkinson's disease and L-DOPA induced dyskinesia.

Keywords: NG2-glia; Parkinson's disease; L-DOPA-induced dyskinesia; Astrocyte; Microglia. MAIN POINTS

- L-DOPA-induced dyskinesia produces a decrease of the NG2-glia density in the striatum, an area critical to Parkinson's disease pathophysiology LID development.

- The antidyskinetic therapy with doxycycline revealed a robust increase of the NG2-glia in the dorsomedial, dorso- and ventrolateral striatum

- NG2-glia has a negative correlation with LID score contrasting to GFAP and OX-42 immunopositive cells.

Abbreviations - 6-OHDA: 6-hydroxydopamine; AIMs: axial, limb and orofacial abnormal involuntary movements; ALO: global AIMs Score including all AIM categories: axial, forelimb, and orofacial; DOXY: doxycycline; GFAP: glial fibrillary acidic protein from astrocyte; L-DOPA: 3,4-dihydroxyphenyl-L-alanine; LID: L-DOPA-induced dyskinesia; NG2-glia: Nerve/glial antigen 2 glial cells; OX-42: CD11b/c equivalent protein of microglia; PD: Parkinson's disease; SNc: substantia nigra compacta; TH: tyrosine hydroxylase.

INTRODUCTION (742 words)

Parkinson's disease (PD) is a neurodegenerative disorder, triggered by the progressive loss of dopamineproducing neurons in the substantia nigra compacta (SNc) region of the basal ganglia (Vaillancourt and Lehericy, 2018). The disease was considered mainly by motor symptomatology, including resting tremor, bradykinesia, limb rigidity, and defects in gait and balance (Obeso et al., 2018). Currently, symptomatic therapies are available, mainly the dopamine replacement therapy with levodopa (L-DOPA: 1-3,4-dihydroxyphenylalanine). L-DOPA improves motor symptoms, but long-term L-DOPA use leads to the gradual development of side effects such as on-off fluctuations, abnormal involuntary movements, and hallucinations (Obeso et al., 2000; Dauer and Przedborski, 2003). The abnormal involuntary movements termed L-DOPA-induced dyskinesia (LID), are among the main challenges in treating PD since they limit the L-DOPA effectiveness.

To date, LID cause is not entirely understood (Fahn et al., 2004; Olanow et al., 2004; Jenner, 2008). Several studies aimed to find alternative therapies that reduce LID (Cenci et al., 2020). It was recently demonstrated the presence of an inflammatory reaction in the brains of patients exhibiting a history of dyskinesia (Del-Bel et al., 2016; Carta et al., 2017; Junior et al., 2020). In the PD post mortem brain patients, the basal ganglia is marked by angiogenesis, vascular endothelial growth factor up-regulation, and altered brain blood barrier properties (Ohlin et al., 2011, 2012; Janelidze et al., 2015; Lerner et al., 2017). Pre-clinical studies showed evidence of an inflammatory environment in the dopamine depleted striatum, with sustained activation of astrocytes and microglia and the recruitment of immune elements contributing to the pathophysiology of LID (Picconi et al., 2002; Robelet et al., 2004; Meissner et al., 2006; Buck and Ferger, 2010; Bortolanza et al., 2015a; Del-Bel et al et al., 2016; Guerra et al., 2019). Supporting the hypothesis, the striatum of lesioned rats long after the microinjection of the neurotoxin 6-hydroxydopamine (6-OHDA), receiving L-DOPA treatment, has been revealed a sustained neuroinflammatory reaction (Spinnewyn et al., 2011; Aron-Badin et al., 2013; Muñoz et al., 2014; Bortolanza et al., 2015a and b; Ramirez-Garcia et al., 2016; Teema et al., al., 2016; Boi et al., 2019). Targeting neuroinflammation may be a strategy to limit LID (Del Bel et al., 2016; Dos-SantosPereira et al., 2016; Carta et al., 2017; Junior et al., 2020).

A population of dividing glial progenitors, called NG2-glia, considered the fourth glial type in the adult central nervous system, has been identified throughout adult brain parenchyma (Dimou et al. 2008; Richardson et al., 2011). This population express markers typically found in oligodendrocyte precursor cells during development 
such as chondroitin sulfate proteoglycan (Levine et al., 1998; Peters, 2004; Nishiyama et al., 2009). It has been reported their potential to generate a wide variety of cell types besides oligodendrocytes, including astrocytes and neurons (Belachew et al., 2003; Aguirre and Gallo, 2004; Aguirre et al., 2010; Baracskay et al., 2007), although the latter is debated (Dimou et al., 2008; Nishiyama et al., 2009; Zhu et al., 2011; Richardson et al., 2011). Rodent NG2 glia receives direct synaptic inputs from glutamatergic and GABAergic neurons, a feature that is unique among glial cells (Bergles, et al., 2000; Lin \& Bergles, 2004). Furthest, it was demonstrated that neuronal activity promotes the recruitment and differentiation of NG2 cells in the adult brain, which could contribute to neural plasticity (Gibson et al., 2014).

NG2-glia reacts to many types of injury or pathological conditions changing their morphology and proliferation rate. Additionally, NG2-glia responds to inflammatory cues, exhibiting a behaviour remarkably similar to microglial cells (Kang et al., 2013; Wang et al., 2017). NG2-glia surveys their microenvironment through constant filopodia extension changing their morphology (Nishiyama et al., 1997; Martín-López et al., 2013; Bribian et al., 2018; Okada et al., 2018). Zhang et al. (2019) described a downregulation of NG2-glia expression in the SNc of PD patient brain compared with healthy subjects. Kitamura et al. (2010) detected activated NG2-positive cells in the nigral 6-OHDA-injected model but not in the striatum. These findings suggest the dysfunction of NG2 glia in the PD brain and provide a compelling rationale for developing new studies.

Here we analyzed the NG2-glia response in the striatum of parkinsonian rats expressing LID using immunoconfocal morphometry, immunohistochemistry and immunoblotting. We examined NG2-glia distribution in the lesioned striatum, the cells' phenotypic characteristics, and the association with astrocytes and microglia cells. Because molecules capable of modulating glial cells' activation were effective in resolving LID, we determine the antidyskinetic effect of doxycycline (6-Deoxy-5-hydroxytetracycline, Bortolanza et al., 2020), in NG2 expression and NG2-glia activation.

METHODS (2.243 words)

Subjects Adult, male Wistar rats ( $\mathrm{n}=64,250-300 \mathrm{~g}$, aged 9-11 weeks) were used in this study. Animals were housed in groups of three per cage, maintained at a temperature of $22-25{ }^{\circ} \mathrm{C}$, on a 12 -h light/dark cycle, and with food and water (autoclaved tap water) available ad libitum. Studies regarding sex differences, which may produce biological variables, were not investigated in this study. The experiments were performed in compliance with the recommendations of the US National Institutes of Health Guide for Care and Use of Laboratory Animals and were approved by the Institutional Animal Care and Use Committee at the University of Sao Paulo (Approval Number: 2017-0014-02). All efforts of the researchers minimized the animal suffering and the number of animals used.

Drugs The dose regimen and route of administration of drugs were based on previously published studies (Cenci et al., 1998; Gomes et al., 2008; Lazzarini et al., 2013; Padovan-Neto et al., 2015). L-DOPA (L-3,4Dihydroxyphenylalanine methyl ester hydrochloride; $20 \mathrm{mg} / \mathrm{kg}$ orally- Prolopa dispersive, Hoffman-LaRoche, Rio de Janeiro, RJ, Brazil), plus benserazide- $\mathrm{HCl}(5 \mathrm{mg} / \mathrm{kg}$ ) were dissolved in water. Doxycycline (40 mg $/ \mathrm{kg}$ i. p., Sigma-Aldrich, St. Louis, MO, USA) was dissolved in saline and administered 30 min before L-DOPA. All drugs and their respective vehicle (VEH) were freshly prepared before use and injected in a volume of $1 \mathrm{~mL} / \mathrm{kg}$.

Parkinsonian Lesion Microinjection of 6-OHDA was delivered into the medial forebrain bundle as previously described (Gomes and Del-Bel, 2003; Gomes et al., 2008; Padovan-Neto et al., 2009). Animals were anaesthetized with 2,2,2-tribromoethanol (Sigma-Aldrich, St. Louis, MO, USA) (250 mg kg-1, i.p.) and fixed into the stereotaxic apparatus for performing the surgery (David Kopf, model USA, 9:57). Stereotaxic coordinates were (from bregma in $\mathrm{mm}$ : $\mathrm{AP}=-4.3 ; \mathrm{LL}=-1.6$; $\mathrm{DV}=-8.3$ ) were based on Paxinos \& Watson, (2007). Rats received microinjection of 6-OHDA in a volume of $2 \mu$ into the left medial forebrain bundle (6-OHDA - $2.5 \mu \mathrm{g} \mu \mathrm{l}-1$ in $0.9 \% \mathrm{NaCl}$ supplemented with $0.02 \%$ ascorbic acid, $1 \mu \mathrm{L}$ min-1). After the microinjection, the cannula was left in place for two additional minutes to prevent the injected solution's reflux. At the end of the surgical procedure, the animals were kept warm by a $60 \mathrm{~W}$ light bulb until full recovery 
from anaesthesia. The dopamine lesion was confirmed by analysis of tyrosine hydroxylase immunoreactivity (TH-ir) as described before (Padovan-Neto et al., 2015) in the striatum and SNc (Fig. 1).

\section{Behavioural tests}

Rotational Behavior After two weeks from surgery, rats were tested for apomorphine-induced rotational behavior $(0.5 \mathrm{mg} / \mathrm{kg}$ s.c. over a period of $45 \mathrm{~min})$. Only animals showing individual mean (2 full turns per min in the direction contralateral to the lesion side) were selected for the study (Padovan-Neto et al. 2009). Two days after that, chronic L-DOPA treatment was initiated.

L-DOPA-Induced dyskinesia Chronic L-DOPA treatment consisted of rats receiving a daily oral administration of L-DOPA for 14 days. Rats were monitored for LID using a rat dyskinesia scale (Anderson et al., 1999; Cenci and Lundblat, 2007; and modified by Padovan-Neto et al., 2009). Briefly, each rat was scored on a severity scale from 0 to 4 (where $0=$ absent, $1=$ occasional, $2=$ frequent, $3=$ continuous but interrupted by sensory distraction, and $4=$ continuous, severe and not suppressible) on each of the three axial, limb, and orofacial (ALO AIMs) subtypes (once every 20 min during 180 min after L-DOPA administration. Results are presented as the sum of ALO AIMs scores and analyzed as one item. Also, A dyskinesia time curve was generated by plotting the L-DOPA induced AIMs score against each monitoring time $(20,40,60,80,100$, $120,140,160$, and $180 \mathrm{~min}$ ) for an entire testing session. Animals with a L-DOPA induced. AIMs score of $>10$ and severity grading of $>2$ on at least one L-DOPA induced AIMs to score subtype were selected for the behavioral analysis (Lundblad et al., 2002; Winkler et al., 2002).

\section{Immunohistochemical analysis of the rat post-mortem tissue}

Tissue preparation. Rats anaesthetized with tribromoethanol (1.5 g kg-1, Sigma-Aldrich, St. Louis, MO, USA) were euthanized 60 min after the last L-DOPA injection. The brain was fixed by transcardiac perfusion with $100 \mathrm{~mL}$ of Kreb's-ringer buffer and $200 \mathrm{~mL}$ of buffered picric acid-paraformaldehyde fixative room temperature (Somogyi and Takagi, $1982-500 \mathrm{ml}$ of $0.2 \mathrm{M}$ sodium phosphate buffer (pH 7.4), $150 \mathrm{ml}$ of saturated picric acid in distilled water; $348 \mathrm{ml}$ of paraformaldehyde solution containing $40 \mathrm{~g}$ of depolymerized paraformaldehyde and $2 \mathrm{ml}$ of $25 \%$ glutaraldehyde). Brains were dissected and post-fixed in the same fixative for 60 minutes at $4{ }^{\circ} \mathrm{C}$. The tissues were equilibrated with $30 \%$ sucrose in $0.1 \mathrm{M}$ phosphate buffer and fully frozen. The brains were quickly frozen in isopentane cooled in liquid nitrogen (-40 degC, Sigma-Aldrich, St. Louis, MO, USA) and stored at $-80 \operatorname{deg} \mathrm{C}$ until histological processing.

Serial coronal sections throughout the rostrocaudal extent of the striatum (Bregma $+2.76 \mathrm{~mm}$, Interaural $11.76 \mathrm{~mm}$ - Bregma $-2.28 \mathrm{~mm}$, Interaural $6.72 \mathrm{~mm}$ ) and the SNc (Bregma $-4.44 \mathrm{~mm}$, Interaural $4.56 \mathrm{~mm}-$ Bregma $-6.24 \mathrm{~mm}$, Interaural $2.76 \mathrm{~mm}$ ) were cut $(25 \mu \mathrm{m})$ using a freezing microtome (Leica, model CM1850).

Tyrosine hydroxylase (TH) Immunoperoxydase Labeling TH immunolabeling to confirm 6-OHDA lesion was detected following a standard peroxidase-based method (Gomes et al., 2008; Padovan-Neto et al., 2009). Sections were incubated overnight at room temperature with the primary antibody: rabbit anti-TH (1:4000, PelFreez, 01,229, USA for rats), followed by $2 \mathrm{~h}$ of incubation with anti-rabbit biotinylated secondary antibody (1:250, Vectastain, Vector Laboratories, USA). Sections were then incubated with the avidin-biotinperoxidase complex for $2 \mathrm{~h}$ (Vectastain ABC kit, Vector Lab, Burlingame, CA, USA). Immunoreactivity was revealed by a peroxidase reaction using 3,3'-Diaminobenzidine diaminobenzidine (DAB; Sigma-Aldrich, St. Louis, MO, USA) as the chromogen. The slices were mounted on slides and coverslipped for microscopic observations.

An in-depth analysis of NG2-glia by immunoconfocal morphometry . Immunofluorescent labeling of NG2glia was achieved incubating brain sections in blocking buffer solution (3\% normal goat serum, $0.05 \%$ Triton $\mathrm{X}-100$ and $1 \%$ bovine serum albumin in phosphate-buffered saline) and then, overnight at $4{ }^{\circ} \mathrm{C}$ with a rabbit polyclonal antibody to NG2 (1:500; Millipore, Temecula, CA, USA). After thorough washing, sections were incubated for 90 min at room temperature in secondary antibodies conjugated to Alexa 488 against rabbit (fluorochrome, 1:1000 Invitrogen). The specificity of primary antibodies immunoreactivity was confirmed by the omission of the primary or secondary antibody and the verification of the absence of immunohistochemical 
staining in these sections. Brain sections were scanned and photographed by slide scanner (SCN400, Leica Microsystems Ltd., Mannheim, Germany) on Multi-channel confocal microscopy (Leica TCS-SP5, Leica Microsystems Ltd., Mannheim, Germany). Digital images were acquired for a wavelength of $488 \mathrm{~nm}$ (green), and the images were converted to TIFF format. Contrast levels adjusted using Adobe Photoshop v. 10.0 (Adobe Systems, San Jose, CA, USA).

Quantitative analysis of the cell number was carried out on the medial striatum dorsal and ventral areas (Bregma $+0.96 \mathrm{~mm}$, Interaural $9.96 \mathrm{~mm}$ - Bregma $-0.24 \mathrm{~mm}$, Interaural $8.76 \mathrm{~mm}-\mathrm{x} 20$ objective) per animal, and results were expressed as an average density of NG2 glia in pixels. Only those on the surface of the sections, where antibody penetration was guaranteed, were counted. Images were digitized with a video camera (Leica DFC420), captured real magnification in grayscale, and evaluated with ImageJ (http:// rsb.info.nih.gov). Integrated optical density values (product of area and mean gray value) of corresponding sectors in each section were averaged. For this procedure, the stain's mean gray value was expressed in arbitrary grayscale units where the scale ranges from 0 to 255 (0 representing the darkest, most intense labeling) to form one density measurement performed in the sections. After this, integrated optical density was calculated by multiplying the selected area with the mean gray value. Sampled areas consisted of regions of interest measuring $0.1 \mathrm{~mm} 2$. The average gray amount from an unstained area was subtracted from each section to correct for background immunoreactivity. All analyses were performed on the lesioned hemisphere of the brain (right) by an experimentally blind investigator.

Quantitative analysis of the ramification index, number of branches, number of junctions, average branch length, and soma area of NG2-glia was carried out on the medial striatum dorsal and ventral areas (Bregma $+0.96 \mathrm{~mm}$, Interaural $9.96 \mathrm{~mm}$ - Bregma $-0.24 \mathrm{~mm}$, Interaural $8.76 \mathrm{~mm}-\mathrm{x} 40$ objective), according to previous studies (Heppner et al., 1998; Eder et al., 1999). Either adjustment to contrast or brightness were made uniformly to all parts of the image. A Sholl analysis method was developed to quantify NG2-glia morphology in immunofluorescent images of the striatum, according to the following: the resulting images were converted to a binary signal and analyzed using the Simple Neurite Tracer plugin of the Fiji software, ImageJ (https://imagej.net/Fiji/Downloads). Cellular branches were manually traced and, the center for the Sholl analysis was pointed at the centroid of the nucleus. Concentric circles were automatically drawn, beginning at $2 \mu \mathrm{m}$ from the center and increasing $2 \mu \mathrm{m}$ with every circle. The Sholl analysis plugin was then applied to all traced cells to collect data on the intersections between branches and each increasing circle to create a Sholl plot. For each rat, a mean Sholl plot was generated. The ramification index (RI) was calculated using the following formula: $\mathrm{RI}=$ cell area/convex area, where "convex area" is the area of a polygonal object defined by the cells' most prominent projections (Schilling and Eder, 2015). The length of NG2-glia processes was defined as the distance between the soma and the detectable end of an extended process identified by NG2 staining. NG2-glia diameter (soma area) was determined by measuring the cell body's longest axis through the nucleus $\left(\mathrm{um}^{2}\right)$.

Dual labeling of NG2-glia and glial fibrillary acidic protein (GFAP, to reveal astrocytes) or OX-42 (CD11b/c equivalent protein of microglia to reveal microglia) was achieved incubating brain sections in blocking buffer solution (3\% normal goat serum, $0.05 \%$ Triton X-100 and $1 \%$ bovine serum albumin in phosphate-buffered saline) and then, overnight at $4{ }^{\circ} \mathrm{C}$ with a rabbit polyclonal antibody to NG2 (1:500; Millipore, Temecula, CA, USA) and mouse monoclonal antibody to GFAP (1:700; Millipore, Temecula, CA, USA) or rabbit polyclonal antibody to NG2 and mouse monoclonal antibody to OX-42 (1:600; Abcam, Branford, CT, USA). After thorough washing, sections were incubated for $90 \mathrm{~min}$ at RT in secondary antibodies conjugated to Alexa 488 against rabbit and Alexa 568 against mouse (fluorochromes, 1:1000 Invitrogen). The specificity of primary antibodies immunoreactivity was confirmed by the omission of the primary or secondary antibody and the verification of the absence of immunohistochemical staining in these sections. Immunofluorescent digital images were obtained using a Leica microscope (Leica Microsystems Launches Leica FW4000 - Cambridge, UK), and were acquired separately for each wavelength [488 nm (green) and $568 \mathrm{~nm}$ (red)] and then merged. The striatal area's quantification was measured using the ImageJ system (National Institutes of Health NIH; Schneider et al., 2012). Striatal NG2, GFAP, and OX-42 immunolabeled cells were quantified at 20X magnification. The striatum was unilaterally evaluated in the dorsolateral, dorsomedial, ventrolateral, and 
ventromedial quadrants. According to the coordinates of the Franklin and Paxinos (1997) rat brain atlas, the regions were located. For double labeling, the results of NG2, GFAP, and OX-42 were analyzed as the number of cells per $0.5 \mathrm{~mm}^{2}$, and they were expressed in percentage of double labeling per total of NG2 cells.

\section{Western blotting (WB) analysis}

Tissue preparation. An independent group of animals was decapitated and the dorsal striatum was dissected on an ice-cooled dissection cover, with the help of magnifying lens (Leica Zoom 2000), and immediately frozen in dry ice. Tissue samples were stored at $-80 \operatorname{deg} C$ until use. The sample was homogenized on ice in 200uL of tissue in sterile saline using a Polytron(r)PT 1200 handheld homogenizer (KinematicaInc; NY, USA). The homogenate was used for WB measurement.

Immunoblotting analysis. Protein was isolated from the striatum of both controls and experimental rats. Samples were treated with boiling lysis buffer (1\% sodium dodecyl sulfate, $1.0 \mathrm{mM}$ sodium orthovanadate, $10 \mathrm{mM}$ Tris, $\mathrm{pH}$ 7.4). Equal amounts $(30 \mu \mathrm{g})$ of total protein were separated by sodium dodecyl sulfate polyacrylamide gel electrophoresis $(10 \%)$ and transferred to polyvinylidene difluoride membranes. Immunostaining of the blots was performed using two primary antibodies, rabbit polyclonal antibody to NG2 (1:1.000; Millipore) and mouse monoclonal antibody to anti- $\beta$-actin (1:10.000; Sigma-Aldrich). Membranes were then incubated with peroxidase-coupled secondary antibodies (1:2.000; Millipore) for 1 hour at room temperature. Blots were developed using the Amersham ECL Prime western blotting detection reagent (GE healthcare, Little Chalfont, UK). Densitometric analysis was performed using the Eagle Eye TMII Still VideoSystem (Stratagene, La Jolla, CA, USA).

\section{Experimental design}

6-OHDA-lesioned rats were randomized to one of the following treatment groups: 6OHDA $(\mathrm{n}=6)$, $6 O H D A+D O X Y(\mathrm{n}=6), 6 O H D A+$ Vehicle $+L-D O P A(\mathrm{n}=7)$, and 6OHDA+DOXY+L-DOPA $(\mathrm{n}=7) . \quad$ ANo lesion group $(\mathrm{n}=6)$, receiving saline in the MFB, was analyzed as a control group. The rats were submitted to analysis of the AIMs as an index of LID. Animals were sacrificed one hour after the last L-DOPA treatment to obtain the striatum for immunohistochemistry/imunoblotting assessment. Rats treated with doxycycline or its vehicle received the drug $30 \mathrm{~min}$ before the last L-DOPA dose. Independent groups of rats submitted to the same treatments and euthanasia were conducted to obtain the striatum for Western blotting methodology.

\section{Statistical analysis}

For the sample calculation, we used the sample size values; critical value for the desired degree of confidence; population standard deviation of the variable; maximum estimated difference between the sample mean and the true population mean (it is the margin of error or maximum error of estimate).

The dyskinesia data failed the Kolmogorov-Smirnov test for normality. Therefore, these results were analyzed by a non-parametric approach. The Mann-Whitney test investigated the differences between treatments.

A one-way ANOVA, followed by Bonferroni's test, was performed to analyze the immunohistochemical and western blotting results.

Spearman's rank correlation coefficient was calculated to investigate associations between the global ALO AIMS and NG2 expression. The statistical analyses were handled using GraphPad Prism software 6 or the Statistical Package for the Social Sciences (SPSS) v.20.0. Data were expressed as the mean \pm standard error of the mean (SEM). Values of $\mathrm{p}<0.05$ were considered statistically significant.

RESULTS (1.073 words)

Nigrostriatal lesion characterization and AIMs scores.

The rate-limiting enzyme in the dopamine synthesis pathway is TH, widely accepted as a marker of dopaminergic neurons. We first examined neurodegeneration in the striatum by TH-immunoreactivity in the brain 
coronal sections of 6-OHDA-lesioned and control animals.

Rats with 6-OHDA lesion exhibited more than 90\% reduction in TH-immunostaining in the striatum and SNc compared to sham lesioned animals (controls; Fig. 1A-D).

In the apomorphine-induced rotational behavior, rats presented strong contralateral turns following apomorphine challenge (results not presented) confirming $>90 \%$ dopaminergic cell loss

In respect to chronic L-DOPA treatment, $90 \%$ of the rats developed AIMs impacting the contralateral forelimb, orofacial movements and axial dystonia (Fig. 1E, p<0.05, 6OHDA+Vehicle+L-DOPA). The maximum total AIMs score was reached 60-120 min after L-DOPA administration and gradually declined to the baseline level over $180 \mathrm{~min}$ (Fig. 1F).

Administration of a single dose of doxycycline (40mg kg-1), $30 \mathrm{~min}$ before L-DOPA, significantly decreased AIMs (79\%; Fig. 1E-F, 6OHDA+Vehicle $+L-D O P A$ AIMs score $=122.41 \pm 24.5$ vs. $6 O H D A+D O X Y+L-D O P A$ ALO AIMs score $=26.77 \pm 10.79, \mathrm{p}<0.001)$ over 20 to $140 \mathrm{~min}$ observation period (Fig. $1 \mathrm{~F})$. AIMs were not observed in both the non-lesioned rats and lesioned rats treated with vehicle or doxycycline (data not shown).

Analysis of NG2-glia in the striatum in 6-OHDA-lesioned Parkinsonian rats treated with L-DOPA by immunoconfocal microscopy

Firstly, we examined the spatial distribution of NG2-glia in the rat striatum of the experimental groups No lesion (control), 6OHDA lesion (6OHDA), 6OHDA+DOXY, 6OHDA+Vehicle+L-DOPA and $6 O H D A+D O X Y+L-D O P A$ (Fig. 2B-E; see methods). The four subdivisions of the striatum (dorsomedial, dorsolateral, ventromedial, ventrolateral) were separately quantified, due to their functional properties, which reflect the diversity of the striatal dopaminergic system connections (Fig. 2F-I).

Parkinsonian rat brains were analyzed 32 days after the neurotoxin microinjection, corresponding to 6 OHDAlesion establishment and L-DOPA/vehicle time therapy. The analysis of the dorsolateral and ventrolateral striatal regions showed a slight increase $(15 \%)$ in NG2-glia density when compared to the No lesion group. This minor increase was maintained after doxycycline treatment (experimental group $6 O H D A+D O X Y$, $\mathrm{p}<0.05$; Fig. $2 \mathrm{G}$ and I).

Parkinsonian rats receiving L-DOPA and reporting LID presented a robust NG2-glia density reduction in the dorsolateral, ventrolateral and dorsomedial striatum when compared to the No lesion (control), 6OH$D A$ lesion (6OHDA), 6OHDA+DOXY and 6OHDA+DOXY+L-DOPA groups (6OHDA+Vehicle+L-DOPA , $\mathrm{p}<0.001$; Fig. 2F, G and I). Doxycycline administration before L-DOPA prevented dyskinesia and lead reduced NG2-glia density in the striatum regions $(6 O H D A+D O X Y+L-D O P A ; \mathrm{p}<0.001 ; \mathrm{Fig}$. $2 \mathrm{~F}, \mathrm{G}$ and I). There was a negative correlation of NG2-glia density with the dyskinesia score (dorsomedial $\mathrm{r}=-0.83$, dorsolateral $\mathrm{r}=-0.88$, and ventrolateral $\mathrm{r}=-0.64 ; \mathrm{p}<0.0001 ; \mathrm{Fig}$. 2J, $\mathrm{K}$ and $\mathrm{M}$ ). There was no NG2-glia immunolabelling alteration in the ventromedial striatum of the animals.

The profile of NG2-glia, analyzed by protein expression immunoblotting in dorsolateral striatum extract, revealed one band of about 26kDa corresponding to NG2-glia in both the control and the lesioned striatum (Fig. 2N). Lesioned rats treated or not with doxycycline showed a slight increase (17\%) in NG2-glia expression when compared to No lesionones (6OHDA and $6 O H D A+D O X Y, \mathrm{p}<0.05$, Fig. 2N). The NG2-glia expression decreased in the lesioned striatum of L-DOPA-treated parkinsonian rats compared to all other groups (50-60\%,6OHDA + Vehicle $+L-D O P A, \mathrm{p}<0.05$, Fig. 2M). Doxycycline treatment induced a significant increase $(76 \%)$ of NG2-glia immunoreactivity compared to the intensity detected in animals presenting LID $(6 O H D A+D O X Y+L-D O P A ; \mathrm{p}<0.05$, Fig. $2 \mathrm{~N})$.

NG2-glia morphological alteration in L-DOPA-induced dyskinesia.

The number of branches, their length $(\mu \mathrm{m})$, the number of junctions and the soma area $\left(\mu \mathrm{m}^{2}\right)$ of NG2-glia were quantified in each of the four subdivisions of striatum (Fig. 3).

Firstly, the ramification index of the NG2-glia after L-DOPA chronic treatment of parkinsonian rats and 
doxycycline administrationincreased in the dorsolateral striatum, decreased in the dorsomedial and ventromedial striata, and presented no changes in the ventrolateral division (Figs A, A", B-E) when compared to rats that received only L-DOPA treatment.

Animals receiving L-DOPA treatment and exhibiting LID presenteda decrease in the number of branches per cell and the average of the branch length in the dorsolateral striatum compared to the 6OHDA lesioned animals. In the dorsomedial and ventromedia 1 striatum L-DOPA treatment-induced, per cell, an increase in the number of branches, the average branches length, and the number of junctions compared to 6 OHDA lesioned animals. The dorsomedial striatumalso showed an increase in the average of the soma area. There was a decrease in NG2-glia cells index activation in the dorsolateral striatum of rats presenting LID (Fig 3).

In the dorsolateral striatum of the animals presenting LID and treated with doxycycline was revealed an increased NG2-glia pattern of activation as evaluated by the number and the average branches length per cell, the number of junctions per cell (entangled with each other) and the average soma area when compared to $6 O H D A$ lesion and6OHDA + Vehicle $+L-D O P A$ groups. There was a decrease in the NG2-glia indicators of activation in the dorsomedial and ventromedial striatum.

Doxycycline treatment decreased dyskinesia and the signals of the NG2-glia activation in the dorsomedial and ventromedial striatum (the number of branches per cell and the average branch length). In the dorsomedial striatum it was also observed a decrease in the NG2-glia average of the soma area compared to $6 O H D A+L-D O P A$ group.

There were no changes in the NG2-glia cells measured parameters in the ventrolateral striatum in all experimental groups (Nolesion (control), 6OHDA lesion, 6OHDA+DOXY, 6OHDA+Vehicle+L-DOPA and $6 O H D A+L-D O P A+D O X Y)$.

Double labelling of NG2-glia and astrocytes or microglia in the striatum of 6-OHDA-lesioned parkinsonian rats treated with L-DOPA and/or doxycycline

We performed a double immunofluorescence reaction to determine the presence and/or co-localization of the astroglial protein GFAP, the microglial protein OX-42 and the NG2-antigen in the striatum from 6OHDA-injected animals and those presenting LID. The yellow pseudo-color for these structures visible in microscopic images identified the superimposition of NG2-glia immunoreactivity (green) on either GFAP or OX-42 positive immunoreactivity (red) cell body/process and vice versa .

Similar to Bortolanza et al., (2015), the dopamine-depleted striatum of rats under L-DOPA treatment $(6 O H D A+$ Vehicle $+L-D O P A, \mathrm{p}<0.001$, Table 1$)$ had a prominent GFAP and OX42 immunoreactivity $(6 O H D A+$ Vehicle $+L-D O P A, \mathrm{p}<0.05$, Table 1). Doxycycline administered before L-DOPA reduced LID and also GFAP and OX42 immunoreactivity in the depleted striatum $(6 O H D A+D O X Y+L-D O P A, \mathrm{p}<0.001$, Table 1).

High resolution in single plane image showed an extensive interdigitation of the processes deriving from astrocytes/microglia and NG2-glia. The fine processes of NG2 (green) and astrocytes (red) or microglia (red), characteristically shown as irregular shaped dots, closely associated in tiny points (yellow) of all subdivisions of striatum (Fig. 4, 5A-D). There was no effect of the lesion or the treatment in the measured parameters of the microglia and astrocytes markers.

DISCUSSION (1.002 words)

Our data revealed (i) a slight increase in the immunoreactivity of NG2-glia in the lesioned dorso- and ventrolateral striatum 36 days after the 6-OHDA-microinjection; (ii) a robust decrease of NG2-glia immunoreactivity in the dorsolateral, dorso- and ventromedial striatum of rats presenting LID with NG2-glia density negatively correlated with LID score; (iii) a robust increase of NG2-glia density in the dorsomedial, dorso- and ventrolateral striatum following doxycycline antidyskinetic therapy, in contrast to a decrease in the GFAP and OX-42 immunoreactivity. The morphometric analysis of the striatum of the animals express- 
ing LID revealed (iv) an increase in the NG2-glia indicators of activation in the dorso- and ventromedial striatum with a decrease in the dorsolateral one. The aforementioned results revealed: (v) a further increase in the activation cells parameters only in the dorsolateral striatum, associated to doxycycline antidyskinetic effect; (vi) a decrease in the NG2-glia indicators of activation in the dorso- and ventromedial striatum. The no-lesion animals and the only 6-OHDA lesioned rats receiving vehicle or doxycycline exhibited: (vii) a NG2-glia morphological features of a typical resting cell; (viii) a NG2-glia immunoreactivity decrease in the dorsal and ventromedial striatum. The results provide evidence of the dynamic involvement of NG2-glia in the dyskinesia induced by L-DOPA chronic treatment of parkinsonian rats.

One of the remarkable findings of the present study is that NG2-glia expression decreases in the dorsal striatum of animals' disclosing LID. The dorsal striatum receives dense dopaminergic innervation from the SNc and the lateral portion of the ventral tegmental area (Haber et al., 2000; Ikemoto, 2007; Lerner et al., 2015), strong projections from somatomotor cortical areas and lateral thalamic motor nuclei (Smith, et al., 2004). Independently, controlled information flows from the SNc to the dorsolateral and dorsomedial striatum (Lerner et al., 2015). The dorsolateral striatum presents a sensorimotor role, controlling voluntary movement severely compromised in PD (Flaherty and Graybiel, 1994).

The doxycycline antidyskinestic action increased NG2-glia cell density, with activated phenotype. In this study, we did not measure L-DOPA plasmatic concentrations. Therefore, a potential doxycycline interference on L-DOPA gastrointestinal absorption cannot be ruled out. However, this is an unlikely possibility because previous research from our group (Bortolanza et al., 2020) provide evidences of no drug interference with the positive motor effects of L-DOPA and the anti-dyskinetic effects of doxycycline were still present when L-DOPA was administered subcutaneously. The large majority of NG2-glia in the adult brain is maintained in a quiescent state under physiological conditions (Hughes et al., 2013). Phenotypically, NG2 glia provides a stereotypic reaction with increased NG2 expression, retraction of cell processes, cell body swelling, cell proliferation, and migration toward the lesion site to almost all kind of injury, independently of the extent of myelin loss (Bedner et al., 2020; Jin et al., 2018; Valny et al., 2018). A neuromodulatory mechanism has been proposed to be related to NG2-glia reactivity (Levine et al., 2016). A co-culture of primary NG2-glia with damage hippocampal slices activated NG2-glia, produced neurotrophic factors and immunomodulatory action by creating anti-inflammatory cytokines as interleukin-10 and transforming growth factor (TGF) $\beta 2$ (Sypecka and Sarnowska, 2014).

PD preclinical studies evidenced NG2-glia as a protector factor against neuroinflammation and dopaminergic neuron apoptosis (Kitamura et al., 2010; Zhang et al., 2019). The NG2-glia ablated mouse brain exhibited increases in pro-inflammatory response subsequent exposure to endotoxin lipopolysaccharide (Zhang et al., 2019). An enriched environment with physical activity induces increased numbers of newborn NG2-positive and GFAP-positive cells in the adult SN and improves motor behavior function in the 6-OHDA rat model of PD (Steiner et al., 2006; Kirby et al., 2019). There are however, contradictory observations. Nakano et al. (2017) and Zhang et al. (2019) found that NG2-glia ablation exacerbated dopaminergic neuronal cell loss in a mouse PD model, induced neurodegeneration, microglia activation, and neuroinflammation in the adult hippocampus. In the post-mortem brain specimens from patients with Alzheimer's disease, there is a reduction in NG2-glia immunoreactivity, which is negatively correlated to microglial immunoreactivity (Nielsen et al., 2013), but associated with the amyloid- $\beta$ plaques (Fiedorowicz et al., 2008; Zhang et al., 2019). As shown in amyotrophic lateral sclerosis models NG2-glia has been observed either to limit central nervous system damage or to actively contribute to neuroinflammation or neurotoxicity (Kang et al., 2013).

Consistent with the notion that NG2-glia, microglia, and astrocytes are distinct glial cells subpopulations NG2/GFAP or NG2/OX42 staining was not once found to be co-localized on the same cell. Noteworthy, the number of NG2/GFAP or NG2/OX42 staining superimposition points was not altered by the factors lesion or treatment. An interaction was demonstrated between NG2 glia and astrocytes (Hamilton et al., 2010; Xu et al., 2014), microglia (Nishiyama et al., 1997), and neurons (Maldonado and Angulo, 2015). This anatomical relationship is a basis for the coupling and functional communication between the glial subtypes. Further, NG2-glia is a source of neurotrophic factors in the central nervous system (Bankston 
et al., 2013). Liu and Aguzzi (2020) demonstrated that NG2-glia and microglia interaction played pivotal roles in regulating microglia states in the adult mouse brain in Alzheimer model disease. Astrocytes signal to NG2-glia via the release of ATP and glutamate that evokes a Ca2+ rise in NG2-glia (Hamilton et al., 2010). Considering that the TGF $\beta$ pathway is essential for normal microglia development (Butovsky et al., 2014; Krasemann et al., 2017), TGF $\beta$ pathway suppression may be associated with microglia dysfunction. Whether the NG2-glia function contributes to the disturbing microglia and astrocyte signature in the dorsal striatum of rats presenting LID is not determined yet.

A limitation of the present study was the performance only in male rats to avoid gender influence in the results. It is important to highlight that the analysis of dyskinesia induced by L-DOPA was originally standardized in female rats (Cenci et al., 1999).

As aforementioned, there is an association between damaged dopamine neurons, L-DOPA-treatment, and dysregulated inflammation (Bortolanza et al., 2015b; Del-Bel et al., 2016; Mulas et al., 2016; Teema et al., 2016). The hypothesis of NG2-glia decrease inflammatory reaction is compatible with the observation of the NG2-glia immunoreactivity decrease in LID, and the increase in the microglia/astrocytes. The implications of this discovery are far-reaching and needs more investigation.

SIGNIFICANCE: Our findings provide the first description of the distribution and morphological changes of NG2-glia in the striatum of parkinsonian rats presenting LID and the effect of doxycycline therapy. They support an inverse link between the transformation of NG2 glia to the reactive form and microglial/astrocyte activation/recruitment in a specific brain region, directly enrolled in PD and the dyskinesia manifestation. These data could reflect the possibility that NG2-glia cells promote striatal plasticity as a form of dyskinesia recovery. Besides, this study may also contribute to establishing NG2-glia as a novel therapeutic target for LID and so, we believe that our findings are of great interest to the neuroscience and medical community.

ACKNOWLEDGMENTS The authors would like to thank Célia A. da-Silva, Sara Saltareli, and Vitor Castania for their technical assistance.

CONFLICT OF INTEREST EADB is listed as co-inventor on a U.S. patent application covering doxycycline and related compounds (Patent No. EP18306400.5. PCT: 1054). All other co-authors declare that no conflict of interest exists.

AUTHOR CONTRIBUTIONS GCN, MB, AB, GCLL performed the experiments. EDB, LLM, GCN, $\mathrm{MB} A B$ contributed to the experimental design. EDB, LLM contributed with resources. GCN, MB, AB, EDB, LLM wrote and edited the manuscript. GCN, MB performed data acquisition and analysis. All authors have approved the final manuscript.

DATA AVAILABILITY STATEMENT: The data that support the findings of this study are available from the corresponding author upon reasonable request.

FUNDING STATEMENT: The study was supported by the Sao Paulo State Foundation for the Support of Research (FAPESP, Brazil; Grants 2014/25029-4 and 2017/24304-0). EDB is a recipient of grants from the National Council for Scientific and Technological Development (CNPq, Brazil). EDB is a CNPq research fellow. MB was recipient of FAPESP, Brazil, fellowship 2016-06602-0). GCN was recipient of FAPESP, Brazil, fellowships 2015/03053-3 and 2018/05146-7 and PNPD CNPQ, 88882.317597/2019-01. LLM: Research Grants from the MICINN (PID2019-105218RB-I00).

\section{REFERENCES}

Aguirre, A., \& Gallo, V. (2004). Postnatal neurogenesis and gliogenesis in the olfactory bulb from NG2expressing progenitors of the subventricular zone. The Journal of neuroscience : the official journal of the Society for Neuroscience, 24(46), 10530-10541. doi: 10.1523/JNEUROSCI.3572-04.2004.

Aguirre, A., Rubio, M. E., \& Gallo, V. (2010). Notch and EGFR pathway interaction regulates neural stem cell number and self-renewal. Nature, 467(7313), 323-327. doi: 10.1038/nature09347. 
Anderson, J. M., Hughes, J. D., Gonzalez, Rothi. L. J., Crucian, G.P., Heilman, K.M. (1999). Developmental stuttering and Parkinson's disease: The effects of levodopa treatment. Journal of Neurology Neurosurgery and Psychiatry, 66, 776-778. doi: 10.1136/jnnp.66.6.776.

Arnett, H. A., Mason, J., Marino, M., Suzuki, K., Matsushima, G. K., Ting, J. P. (2001). TNF alpha promotes proliferation of oligodendrocyte progenitors and remyelination. Nature Neuroscience, 4, 1116-22. doi: $10.1038 / \mathrm{nn} 738$.

Aron Badin, R., Spinnewyn, B., Gaillard, M.C., Jan, C., Malgorn, C., van Camp, N., et al. (2013). IRC082451, a Novel Multitargeting Molecule, Reduces L-DOPA-Induced Dyskinesias in MPTP Parkinsonian Primates. PLoS ONE, 8, e52680. doi: 10.1371/journal.pone.0052680.

Bankston, A. N., Mandler, M. D., Feng, Y. (2013). Oligodendroglia and neurotrophic factors in neurodegeneration. Neurosci Bull, 29, 216-218. doi: 10.1007/s12264-013-1321-3.

Baracskay, K. L., Kidd, G. J., Miller, R. H., Trapp, B. D. (2007). NG2-positive cells generate A2B5-positive oligodendrocyte precursor cells. Glia, 55, 1001-10. doi: 10.1002/glia.20519.

Barriola, S., Perez-Cerda, F., Matute, C., Bribian, A., \& Lopez-Mascaraque, L. (2020). A Clonal NG2-Glia Cell Response in a Mouse Model of Multiple Sclerosis. Cells, 9(5), 1279. https://doi.org/10.3390/cells9051279Bedner, P., Jabs, R., Steinhauser, C. (2020). Properties of human astrocytes and NG2 glia. Glia, 68(4):756-767. 10.1002/glia.23725.

Belachew, S., Chittajallu, R., Aguirre, A. A., Yuan, X., Kirby, M., Anderson, S., \& Gallo, V. (2003). Postnatal NG2 proteoglycan-expressing progenitor cells are intrinsically multipotent and generate functional neurons. The Journal of cell biology, 161(1), 169-186. doi: 10.1083/jcb.200210110.

Bergles, D. E., Roberts, J. D. B., Somogyi, P., \& Jahr, C. E. (2000). Glutamatergic synapses on oligodendrocyte precursor cells in the hippocampus. Nature, 405(6783), 187-191. https://doi.org/10.1038/ 35012083;

Boi, L., Pisanu, A., Greig, N.H., Scerba, M.T., Tweedie, D., Mulas, G., Fenu, S., Carboni, E., Spiga, S., Carta, A.R., 2019. Immunomodulatory drugs alleviate l-dopa-induced dyskinesia in a rat model of Parkinson's disease. Movement Disorders, 34, 1818-1830. doi: 10.1002/mds.27799.

Bortolanza, M., Cavalcanti-Kiwiatkoski, R., Padovan-Neto, F. E., da-Silva, C. A., Mitkovski, M., RaismanVozari, R., \& Del-Bel, E. (2015a). Glial activation is associated with l-DOPA induced dyskinesia and blocked by a nitric oxide synthase inhibitor in a rat model of Parkinson's disease. Neurobiology of disease, 73, 377-387. https://doi.org/10.1016/j.nbd.2014.10.017

Bortolanza, M., Nascimento, G.C., Raisman-Vozari, R., Del Bel, E.A. (2020). Preprint- Doxycycline, an anti-inflammatory agent, alleviates dyskinesia induced byL-DOPA in Parkinsonian Rats. June2020. doi: 10.22541 .

Bortolanza, M., Padovan-Neto, F.E., Cavalcanti-Kiwiatkoski, R., Dos Santos-Pereira, M., Mitkovski, M., Raisman-Vozari, R., Del-Bel, E. (2015b). Are cyclooxygenase-2 and nitric oxide involved in the dyskinesia of parkinson's disease induced by L-DOPA? Philosophical Transactions of the Royal Society B: Biological Sciences, 370, 20140190. doi: 10.1098/rstb.2014.0190.

Bribian, A., Perez-Cerda, F., Matute, C., \& Lopez-Mascaraque, L. (2018). Clonal Glial Response in a Multiple Sclerosis Mouse Model. Frontiers in cellular neuroscience, 12, 375. https://doi.org/10.3389/fncel.2018.00375

Buck, K., Voehringer, P., Ferger, B. (2010). The alpha(2) adrenoceptor antagonist idazoxan alleviates L-DOPA-induced dyskinesia by reduction of striatal dopamine levels: an in vivo microdialysis study in 6-hydroxydopamine-lesioned rats. Journal of Neurochemistry, 112, 444-52. doi: 10.1111/j.14714159.2009.06482.x. 
Butovsky, O., Jedrychowski, M. P., Moore, C. S., Cialic, R., Lanser, A. J., Gabriely, G., Koeglsperger, T., Dake, B., Wu, P. M., Doykan, C. E., Fanek, Z., Liu, L., Chen, Z., Rothstein, J. D., Ransohoff, R. M., Gygi, S. P., Antel, J. P., \& Weiner, H. L. (2014). Identification of a unique TGF- $\beta$-dependent molecular and functional signature in microglia. Nature neuroscience, 17(1), 131-143. doi: 10.1038/nn.3599.

Carta, A.R., Mulas, G., Bortolanza, M., Duarte, T., Pillai, E., Fisone, G., Vozari, R.R., Del-Bel, E. (2017). l-DOPA-induced dyskinesia and neuroinflammation: do microglia and astrocytes play a role? Euroupean Journal of Neuroscience, 45, 73-91. doi: 10.1111/ejn.13482.

Cenci, M.A., Lee, C.S., Björklund, A. (1998). L-DOPA-induced dyskinesia in the rat is associated with striatal overexpression of prodynorphin- and glutamic acid decarboxylase mRNA. European Journal of Neuroscience, 10, 2694-2706. PMID: 9767399.

Cenci, M.A., Lundblad, M. (2007). Ratings of L-DOPA-induced dyskinesia in the unilateral 6-OHDA lesion model of Parkinson's disease in rats and mice. Curr Protoc Neurosci. Chapter 9: Unit 9.25. doi: 10.1002/0471142301.ns0925s41.

Cenci, M.A., Riggare, S., Pahwa, R., Eidelberg, D., Hauser, R.A. (2020). Dyskinesia matters. Movement Disorders, 35, 392-396. doi: 10.1002/mds.27959.

Chew, L. J., King, W. C., Kennedy, A., \& Gallo, V. (2005). Interferon-gamma inhibits cell cycle exit in differentiating oligodendrocyte progenitor cells. Glia, 52(2), 127-143. https://doi.org/10.1002/glia.20232.

Dauer W., Przedborski S. (2003). Parkinson's disease: mechanisms and models. Neuron 39, 88990910.1016/S0896-6273(03)00568-3.

Del-Bel, E., Bortolanza, M., Dos-Santos-Pereira, M., Bariotto, K., Raisman-Vozari, R. (2016). L-DOPAinduced dyskinesia in Parkinson's disease: Are neuroinflammation and astrocytes key elements? Synapse, 70, 479-500. doi: 10.1002/syn.21941.

Dimou, L., \& Götz, M. (2014). Glial cells as progenitors and stem cells: new roles in the healthy and diseased brain. Physiological reviews, 94(3), 709-737. https://doi.org/10.1152/physrev.00036.2013

Dimou, L., Simon, C., Kirchhoff, F., Takebayashi, H., \& Götz, M. (2008). Progeny of Olig2-expressing progenitors in the Gray and white matter of the adult mouse cerebral cortex. Journal of Neuroscience, 28(41),10434-10442. https://doi.org/10.1523/JNEUROSCI.2831-08.2008

dos-Santos-Pereira, M., da-Silva, C.A., Guimarães, F.S., Del-Bel, E. (2016). Co-administration of cannabidiol and capsazepine reduces L-DOPA-induced dyskinesia in mice: Possible mechanism of action. Neurobiology of Disease, 94, 179-95. doi: 10.1016/j.nbd.2016.06.013.

Eder, C., Schilling, T., Heinemann, U., Haas, D., Hailer, N., \& Nitsch, R. (1999). Morphological, immunophenotypical and electrophysiological properties of resting microglia in vitro. The European Journal of Neuroscience, 11(12), 4251-4261. doi: 10.1046/j.1460-9568.1999.00852.x

Fahn, S., Oakes, D., Shoulson, I., Kieburtz, K., Rudolph, A., Lang, A., Olanow, C.W., Tanner, C., Marek, K. (2004). Parkinson Study Group. Levodopa and the progression of Parkinson's disease. New England Journal of Medicine. 351, 2498-508. doi: 10.1056/NEJMoa033447.

Fiedorowicz, A., Figiel, I., Zaremba, M., Dzwonek, K., Oderfeld-Nowak, B. (2008). The ameboid phenotype of NG2 (+) cells in the region of apoptotic dentate granule neurons in trimethyltin intoxicated mice shares antigen properties with microglia/macrophages. Glia, 56, 209-22. doi: 10.1002/glia.20605.

Flaherty, A. W., \& Graybiel, A. M. (1994). Input-output organization of the sensorimotor striatum in the squirrel monkey. Journal of Neuroscience, 14, 599-610. doi: 10.1523/JNEUROSCI.14-02-00599.1994.

Gibson, E. M., Purger, D., Mount, C. W., Goldstein, A. K., Lin, G. L., Wood, L. S., Inema, I., Miller, S. E., Bieri, G., Zuchero, J. B., Barres, B. A., Woo, P. J., Vogel, H., \& Monje, M. (2014). Neuronal activity 
promotes oligodendrogenesis and adaptive myelination in the mammalian brain. Science 344(6183), 1252304. doi: $10.1126 /$ science.1252304.

Gomes, M. Z., Del Bel, E. A. (2003). Effects of electrolytic and 6-hydroxydopamine lesions of rat nigrostriatal pathway on nitric oxide synthase and nicotinamide adenine dinucleotide phosphate diaphorase. Brain Research Bulletin, 62, 107-15. doi: 10.1016/j.brainresbull.2003.08.010.

Gomes, M. Z., Raisman-Vozari, R., Del Bel, E. A. (2008). A nitric oxide synthase inhibitor decreases 6hydroxydopamine effects on tyrosine hydroxylase and neuronal nitric oxide synthase in the rat nigrostriatal pathway. Brain Research. 1203, 160-169. doi: 10.1016/j.brainres.2008.01.088.

Haber, S. N., Fudge, J. L., \& McFarland, N. R. (2000). Striatonigrostriatal pathways in primates form an ascending spiral from the shell to the dorsolateral striatum. The Journal of Neuroscience : the official journal of the Society for Neuroscience, 20(6), 2369-2382. doi: 10.1523/JNEUROSCI.20-06-02369.2000.

Hamilton, N., Vayro, S., Wigley, R., \& Butt, A. M. (2010). Axons and astrocytes release ATP and glutamate to evoke calcium signals in NG2-glia. Glia, 58(1), 66-79. doi:10.1002/glia.20902

Hamilton, N., Vayro, S., Wigley, R., Butt, A. M. (2010). Axons and astrocytes release ATP and glutamate to evoke calcium signals in NG2-glia. Glia, 58, 66-79. doi: 10.1002/glia.20902. PMID: 19533604.

Heppner, F. L., Roth, K., Nitsch, R., \& Hailer, N. P. (1998). Vitamin E induces ramification and downregulation of adhesion molecules in cultured microglial cells. Glia, 22(2), 180-188. PMID: 9537838.

Horner, P. J., Thallmair, M., \& Gage, F. H. (2002). Defining the NG2-expressing cell of the adult CNS. Journal of neurocytology, 31(6-7), 469-480. https://doi.org/10.1023/a:1025739630398

Hughes, E. G., Kang, S. H., Fukaya, M., \& Bergles, D. E. (2013). Oligodendrocyte progenitors balance growth with self-repulsion to achieve homeostasis in the adult brain. Nature neuroscience, 16(6), 668-676. doi: $10.1038 / \mathrm{nn} .3390$.

Ikemoto S. (2007). Dopamine reward circuitry: two projection systems from the ventral midbrain to the nucleus accumbens-olfactory tubercle complex. Brain Research reviews, 56(1), 27-78. doi: 10.1016/j.brainresrev.2007.05.004.

Janelidze, S., Lindqvist, D., Francardo, V., Hall, S., Zetterberg, H., Blennow, K., Adler, C.H., Beach, T.G., Serrano, G.E., van Westen, D., Londos, E., Cenci, M.A., Hansson, O. (2015). Increased CSF biomarkers of angiogenesis in Parkinson disease. Neurology, 85, 1834-42. doi: 10.1212/WNL.0000000000002151.

Jenner, P. (2008). Molecular mechanisms of L-DOPA-induced dyskinesia. Nature Reviews Neuroscience, 9, 665-77. doi: 10.1038/nrn2471.

Jin, X., Riew, T. R., Kim, H. L., Choi, J. H., \& Lee, M. Y. (2018). Morphological characterization of NG2 glia and their association with neuroglial cells in the 3-nitropropionic acid-lesioned striatum of rat. Scientific Reports, 8(1), 5942. doi: 10.1038/s41598-018-24385-0.

Kang, S. H., Li, Y., Fukaya, M., Lorenzini, I., Cleveland, D. W., Ostrow, L. W., Rothstein, J. D., \& Bergles, D. E. (2013). Degeneration and impaired regeneration of gray matter oligodendrocytes in amyotrophic lateral sclerosis. Nature Neuroscience, 16(5), 571-579. doi: 10.1038/nn.3357.

Kirby, L., Jin, J., Cardona, J. G., Smith, M. D., Martin, K. A., Wang, J., Strasburger, H., Herbst, L., Alexis, M., Karnell, J., Davidson, T., Dutta, R., Goverman, J., Bergles, D., \& Calabresi, P. A. (2019). Oligodendrocyte precursor cells present antigen and are cytotoxic targets in inflammatory demyelination. Nature Communications, 10(1), 3887. doi: 10.1038/s41467-019-11638-3.

Kitamura, Y., Inden, M., Minamino, H., Abe, M., Takata, K., \& Taniguchi, T. (2010). The 6hydroxydopamine-induced nigrostriatal neurodegeneration produces microglia-like NG2 glial cells in the rat substantia nigra. Glia, 58(14), 1686-1700. doi: 10.1002/glia.21040. 
Krasemann, S., Madore, C., Cialic, R., Baufeld, C., Calcagno, N., El Fatimy, R., Beckers, L., O'Loughlin, E., Xu, Y., Fanek, Z., Greco, D. J., Smith, S. T., Tweet, G., Humulock, Z., Zrzavy, T., Conde-Sanroman, P., Gacias, M., Weng, Z., Chen, H., Tjon, E., .. Butovsky, O. (2017). The TREM2-APOE Pathway Drives the Transcriptional Phenotype of Dysfunctional Microglia in Neurodegenerative Diseases. Immunity, 47(3), 566-581.e9. doi: 10.1016/j.immuni.2017.08.008.

Lazzarini, M., Martin, S., Mitkovski, M., Vozari, R.R., Stühmer, W., Bel Del, E. (2013). Doxycycline restrains glia and confers neuroprotection in a 6-OHDA Parkinson model. Glia, 61, 1084-100. doi: 10.1002/glia.22496.

Lerner, R.P., Francardo, V., Fujita, K., Bimpisidis, Z., Jourdain, V.A., Tang, C.C., Dewey, S.L., Chaly, T., Cenci, M.A., Eidelberg, D. (2017). Levodopa-induced abnormal involuntary movements correlate with altered permeability of the blood-brain-barrier in the basal ganglia. Scientific Reports, 7, 16005. doi: 10.1038/s41598017-16228-1.

Lerner, T. N., Shilyansky, C., Davidson, T. J., Evans, K. E., Beier, K. T., Zalocusky, K. A., Crow, A. K., Malenka, R. C., Luo, L., Tomer, R., \& Deisseroth, K. (2015). Intact-Brain Analyses Reveal Distinct Information Carried by SNc Dopamine Subcircuits. Cell, 162(3), 635-647. doi: 10.1016/j.cell.2015.07.014.

Levine J. (2016). The reactions and role of NG2 glia in spinal cord injury. Brain Research, 1638(Pt B), 199-208. doi: 10.1016/j.brainres.2015.07.026

Levine, J. M., Enquist, L. W., \& Card, J. P. (1998). Reactions of oligodendrocyte precursor cells to alpha herpesvirus infection of the central nervous system. Glia, 23(4), 316-328. PMID: 9671962.

Lin, S., \& Bergles, D. E. (2004). Synaptic signaling between GABAergic interneurons and oligodendrocyte precursor cells in the hippocampus. Nature Neuroscience. 7(1), 24-32. doi: 10.1038/nn1162.

Liu, Y., \& Aguzzi, A. (2020). NG2 glia are required for maintaining microglia homeostatic state. Glia, 68, 345-355. doi: 10.1002/glia.23721.

Lundblad, M., Andersson, M., Winkler, C., Kirik, D., Wierup, N., Cenci, M.A. (2002). Pharmacological validation of behavioural measures of akinesia and dyskinesia in a rat model of Parkinson's disease. European Journal of Neuroscience, 15, 120-32. doi: 10.1046/j.0953-816x.2001.01843. x.

Maldonado, P. P., \& Angulo, M. C. (2015). Multiple Modes of Communication between Neurons and Oligodendrocyte Precursor Cells. The Neuroscientist 21(3), 266-276. doi: 10.1177/1073858414530784.

Martín-López, E., García-Marques, J., Núñez-Llaves, R., \& López-Mascaraque, L. (2013). Clonal astrocytic response to cortical injury. PloS one, 8(9), e74039. doi: 10.1371/journal.pone.0074039.

Meissner, W., Ravenscroft, P., Reese, R., Harnack, D., Morgenstern, R., Kupsch, A., Klitgaard, H., Bioulac, B., Gross, C. E., Bezard, E., Boraud, T. (2006). Increased slow oscillatory activity in substantia nigra pars reticulata triggers abnormal involuntary movements in the 6-OHDA-lesioned rat in the presence of excessive extracellular striatal dopamine. Neurobiology of Disease, 22, 586-98. doi: 10.1016/j.nbd.2006.01.009.

Mulas, G., Espa, E., Fenu, S., Spiga, S., Cossu, G., Pillai, E., et al. (2016). Differential induction of dyskinesia and neuroinflammation by pulsatile versus continuous L-DOPA delivery in the 6-OHDA model of Parkinson's disease. Experimental Neurology, 286, 83-92. doi: 10.1016/j.expneurol.2016.09.013.

Muñoz, A., Garrido-Gil, P., Dominguez-Meijide, A., Labandeira-Garcia, J.L. (2014). Angiotensin type 1 receptor blockage reduces l-dopa-induced dyskinesia in the 6-OHDA model of Parkinson's disease. Involvement of vascular endothelial growth factor and interleukin-1 $\beta$. Experimental Neurology, 261, 720-432. doi: 10.1016/j.expneurol.2014.08.019.

Nakano, M., Tamura, Y., Yamato, M., Kume, S., Eguchi, A., Takata, K., Watanabe, Y., \& Kataoka, Y. (2017). NG2 glial cells regulate neuroimmunological responses to maintain neuronal function and survival. Scientific reports, 7, 42041. doi: 10.1038/srep42041. 
Nielsen, H. M., Ek, D., Avdic, U., Orbjörn, C., Hansson, O., Netherlands Brain Bank, Veerhuis, R., Rozemuller, A. J., Brun, A., Minthon, L., \& Wennström, M. (2013). NG2 cells, a new trail for Alzheimer's disease mechanisms?. Acta neuropathologica communications, 1(1), 7. doi: 10.1186/2051-5960-1-7.

Nishiyama, A., Komitova, M., Suzuki, R., \& Zhu, X. (2009). Polydendrocytes (NG2 cells): multifunctional cells with lineage plasticity. Nature reviews. Neuroscience, 10(1), 9-22. doi: 10.1038/nrn2495

Nishiyama, A., Yu, M., Drazba, J. A., \& Tuohy, V. K. (1997). Normal and reactive NG2+ glial cells are distinct from resting and activated microglia. Journal of neuroscience research, 48(4), 299-312. doi: 10.1002/(sici)1097-4547(19970515)48:4<299::aid-jnr2>3.0.co;2-6.

Nishiyama, A., Yu, M., Drazba, J. A., Tuohy, V. K. (1997). Normal and reactive NG2+ glial cells are distinct from resting and activated microglia. Journal of Neuroscience Research, 48, 299-312. doi: 10.1002/(sici)10974547(19970515)48:4<299::aid-jnr2>3.0.co;2-6.

Obeso, J. A., Olanow, C. W., Nutt, J.G. (2000). Levodopa motor complications in Parkinson's disease. Trends Neuroscience, 23, S2-7. doi: 10.1016/s1471-1931(00)00031-8. PMID: 11052214.

Obeso, J. A., Stamelou, M., Goetz, C. G., Poewe, W., Lang, A. E., Weintraub, D., Burn, D., Halliday, G. M., Bezard, E., Przedborski, S., Lehericy, S., Brooks, D. J., Rothwell, J. C., Hallett, M., DeLong, M. R., Marras, C., Tanner, C. M., Ross, G. W., Langston, J. W., Klein, C., .. Stoessl, A. J. (2017). Past, present, and future of Parkinson's disease: A special essay on the 200th Anniversary of the Shaking Palsy. Movement disorders : official journal of the Movement Disorder Society, 32(9), 1264-1310. https://doi.org/10.1002/mds.27115.

Ohlin, K. E., Francardo, V., Lindgren, H.S., Sillivan, S. E., O’Sullivan, S. S., Luksik, A. S., Vassoler, F. M., Lees, A. J., Konradi, C., Cenci, M. A. (2011). Vascular endothelial growth factor is upregulated by L-dopa in the parkinsonian brain: implications for the development of dyskinesia. Brain. 134(Pt 8): 2339-57. doi: $10.1093 /$ brain/awr165.

Ohlin, K. E., Sebastianutto, I., Adkins, C. E., Lundblad, C., Lockman, P. R., Cenci, M. A. (2012). Impact of L-DOPA treatment on regional cerebral blood flow and metabolism in the basal ganglia in a rat model of Parkinson's disease. Neuroimage, 61, 228-39. doi: 10.1016/j.neuroimage.2012.02.066.

Okada, S., Hara, M., Kobayakawa, K., Matsumoto, Y., Nakashima, Y. (2018). Astrocyte reactivity and astrogliosis after spinal cord injury. Neuroscience Research, 126, 39-43. doi: 10.1016/j.neures.2017.10.004.

Olanow, C. W., Agid, Y., Mizuno, Y., Albanese, A., Bonuccelli, U., Damier, P., De Yebenes, J., Gershanik, O., Guttman, M., Grandas, F., Hallett, M., Hornykiewicz, O., Jenner, P., Katzenschlager, R., Langston, W.J., LeWitt, P., Melamed, E., Mena, M. A., Michel, P. P., Mytilineou, C., Obeso, J. A., Poewe, W., Quinn, N., Raisman-Vozari, R., Rajput, A. H., Rascol, O., Sampaio, C., Stocchi, F. (2004). Levodopa in the treatment of Parkinson's disease: current controversies. Movement Disorders, 19, 997-1005. doi: 10.1002/mds.20243.

Padovan-Neto, F. E., Cavalcanti-Kiwiatkoviski, R., Carolino, R. O. G., Anselmo-Franci, J., Del Bel, E. (2015). Effects of prolonged neuronal nitric oxide synthase inhibition on the development and expression of l-DOPA-induced dyskinesia in 6-OHDA-lesioned rats. Neuropharmacology, 89, 87-97. doi: 10.1016/j.neuropharm.2014.08.019 0028-3908.

Padovan-Neto, F.E., Echeverry, M.B., Tumas, V., Del-Bel, E.A. (2009). Nitric oxide synthase inhibition attenuates l-DOPA-induced dyskinesias in a rodent model of Parkinson's disease. Neuroscience, 159, 927-35. doi: 10.1016/j.neuroscience.2009.01.034.

Paxinos, G., Watson, C. (2004). The Rat Brain in Stereotaxic Coordinates - The New Coronal Set. Elsevier.

Peters A. (2004). A fourth type of neuroglial cell in the adult central nervous system. Journal of neurocytology, 33(3), 345-357. doi: 10.1023/B:NEUR.0000044195.64009.27.

Picconi, B., Bagetta, V., Ghiglieri, V., Paillè, V., Di Filippo, M., Pendolino, V., Tozzi, A., Giampà, C., Fusco, F.R., Sgobio, C., Calabresi, P. (2011). Inhibition of phosphodiesterases rescues striatal long-term depression 
and reduces levodopa-induced dyskinesia. Brain, 134, 375-87. doi: 10.1093/brain/awq342.

Picconi, B., Centonze, D., Håkansson, K., Bernardi, G. (2003). Greengard P, Fisone G, Cenci MA, Calabresi P. Loss of bidirectional striatal synaptic plasticity in L-DOPA-induced dyskinesia. Nature Neuroscience, 6 , 501-6. doi: 10.1038/nn1040.

Ramírez-García, G., Palafox-Sánchez, V., Limón, I.D. (2015). Nitrosative and cognitive effects of chronic L-DOPA administration in rats with intra-nigral 6-OHDA lesion. Neuroscience. 290, 492-508. doi: 10.1016/j.neuroscience.2015.01.047.

Richardson, W. D., Young, K. M., Tripathi, R. B., McKenzie, I. (2011). NG2-glia as multipotent neural stem cells: fact or fantasy? Neuron, 70, 661-73. doi: 10.1016/j.neuron.2011.05.013.

Robelet, S., Melon, C., Guillet, B., Salin, P., Kerkerian-Le, G. L. (2004). Chronic L-DOPA treatment increases extracellular glutamate levels and GLT1 expression in the basal ganglia in a rat model of Parkinson's disease. European Journal of Neuroscience, 20,1255-66. doi: 10.1111/j.1460-9568.2004.03591.x.

Schilling, T., \& Eder, C. (2015). Microglial K(+) channel expression in young adult and aged mice. Glia, 63(4), 664-672. doi: 10.1002/glia.22776

Schneider, C. A., Rasband, W. S., \& Eliceiri, K. W. (2012). NIH Image to ImageJ: 25 years of image analysis. Nature methods, 9(7), 671-675. doi: 10.1038/nmeth.2089.

Smith, Y., Raju, D. V., Pare, J. F., Sidibe, M. (2004). The thalamostriatal system: a highly specific network of the basal ganglia circuitry. Trends Neuroscience, 27, 520-7. doi: 10.1016/j.tins.2004.07.004.

Spinnewyn, B., Charnet, C., Cornet, S., Roubert, V., Chabrier, P. E., Auguet, M. (2010). An improved model to investigate the efficacy of antidyskinetic agents in hemiparkinsonian rats. Fundamental \& Clinical Pharmacology, 25, 608-18. doi: 10.1111/j.1472-8206.2010.00883.x.

Steiner, B., Winter, C., Hosman, K., Siebert, E., Kempermann, G., Petrus, D. S., \& Kupsch, A. (2006). Enriched environment induces cellular plasticity in the adult substantia nigra and improves motor behavior function in the 6-OHDA rat model of Parkinson's disease. Experimental Neurology, 199(2), 291-300. doi: 10.1016/j.expneurol.2005.11.004

Sypecka, J., \& Sarnowska, A. (2014). The neuroprotective effect exerted by oligodendroglial progenitors on ischemically impaired hippocampal cells. Molecular Neurobiology, 49(2), 685-701. doi: 10.1007/s12035-0138549-9

Teema, A. M., Zaitone, S. A., Moustafa, Y. M. (2016). Ibuprofen or piroxicam protects nigral neurons and delays the development of l-dopa induced dyskinesia in rats with experimental Parkinsonism: Influence on angiogenesis. Neuropharmacology, 107, 432-450. doi: 10.1016/j.neuropharm.2016.03.034.

Vaillancourt, D. E., \& Lehericy, S. (2018). Illuminating basal ganglia and beyond in Parkinson's disease. Movement Disorders 33(9), 1373-1375. doi: 10.1002/mds.27483

Valny, M., Honsa, P., Waloschkova, E., et al. (2018). A single-cell analysis reveals multiple roles of oligodendroglial lineage cells during post-ischemic regeneration. Glia, 66(5), 1068-1081. doi: 10.1002/glia.23301.

Wang, C., Zhang, C. J., Martin, B. N., Bulek, K., Kang, Z., Zhao, J., Bian, G., Carman, J. A., Gao, J., Dongre, A., Xue, H., Miller, S. D., Qian, Y., Hambardzumyan, D., Hamilton, T., Ransohoff, R. M., \& Li, X. (2017). IL-17 induced NOTCH1 activation in oligodendrocyte progenitor cells enhances proliferation and inflammatory gene expression. Nature Communications, 8, 15508. doi: 10.1038/ncomms15508

Wennström M, Hellsten J, Tingström A. (2004). Electroconvulsive seizures induce proliferation of NG2-expressing glial cells in adult rat amygdala. Biological Psychiatry, 55, 464-71. doi: 10.1016/j.biopsych.2003.11.011. 
Winkler, C., Kirik, D., Björklund, A., \& Cenci, M. A. (2002). L-DOPA-induced dyskinesia in the intrastriatal 6-hydroxydopamine model of parkinson's disease: relation to motor and cellular parameters of nigrostriatal function. Neurobiology of Disease, 10(2), 165-186. doi: 10.1006/nbdi.2002.0499

Winkler, C., Kirik, D., Björklund, A., Cenci, M.A. (2002). L-DOPA-induced dyskinesia in the intrastriatal 6-hydroxydopamine model of Parkinson's disease: Relation to motor and cellular parameters of nigrostriatal function. Neurobiology of Disease, 10, 165-86. doi: 10.1006/nbdi.2002.0499.

Xu, G., Wang, W., \& Zhou, M. (2014). Spatial organization of NG2 glial cells and astrocytes in rat hippocampal CA1 region. Hippocampus, 24(4), 383-395. doi: 10.1002/hipo.22232

Zhang, S. Z., Wang, Q. Q., Yang, Q. Q., Gu, H. Y., Yin, Y. Q., Li, Y. D., Hou, J. C., Chen, R., Sun, Q. Q., Sun, Y. F., Hu, G., \& Zhou, J. W. (2019). NG2 glia regulate brain innate immunity via TGF- $\beta 2 / T G F B R 2$ axis. BMC medicine, 17(1), 204. doi: 10.1186/s12916-019-1439-x.

Zhu, X., Hill, R. A., Dietrich, D., Komitova, M., Suzuki, R., \& Nishiyama, A. (2011). Age-dependent fate and lineage restriction of single NG2 cells. Development (Cambridge, England), 138(4), 745-753. doi: 10.1242/dev.047951.

Table 1. GFAP and OX-42 expression in DL striatum by immunohistochemistry

\begin{tabular}{llll}
\hline & GFAP-ir cells/ & GFAP-ir cells/ & \\
TREATMENTS & 0.5mm & OX-42-ir cells/ $0.5 \mathrm{~mm}^{2}$ \\
\hline No lesion & No lesion & $0.5 \mathrm{~mm}^{2}$ & $67.7 \pm 12.1^{* \#}$ \\
6-OHDA & 6-OHDA & $112.7 \pm 11.4^{*}$ & $387.3 \pm 3.2^{*}$ \\
6-OHDA+DOXY & 6-OHDA+DOXY & $127.5 \pm 8.7$ & $195.4 \pm 12.6$ \\
6-OHDA+Vehicle & 6-OHDA+Vehicle & $342.2 \pm 24.9^{*}$ & $629.8 \pm 16.2^{*}$ \\
+L-DOPA & +L-DOPA & & \\
6-OHDA+DOXY+L- & 6-OHDA+DOXY+L- & $258.1 \pm 29.2^{*}$ & $388.5 \pm 7.5^{*}$ \\
DOPA & DOPA & & \\
\hline
\end{tabular}

${ }^{*} \mathrm{P}<0.001$ (One-Way ANOVA), vs. 6OHDA+LOPA. ${ }^{*} \mathrm{P}<0.05$, vs. 6OHDA. Data are mean \pm SEM. DOXY: doxycycline; L-DOPA, L-3, 4-dihydroxyphenylalanine; 6-OHDA, 6-hydroxydopamine.

\section{FIGURE LEGENDS}

Figure 1. 6-OHDA nigrostriatal lesion characterization and AIMs scores. Panel A shows the distribution of $\mathrm{TH}$ fibers in the striatum $(\mathrm{CPu})$ of controls (No-lesion- $\mathbf{A}$ and the inset) shows intense THimmunoreactive fiber terminals. Panel $\mathbf{B}$ shows the effect of 6-OHDA on TH-immunoreactive fiber terminals. Likewise in the SNc, TH-immunoreactive neurons were greatly reduced (D) compared to controls (C). Panels $\mathbf{E}$ and Frepresent the effects of doxycycline on the development of abnormal involuntary movements (AIMs $\mathbf{E})$ and time course of AIMs during $180 \mathrm{~min}(\mathbf{F})$ induced by chronic administration of L-DOPA to 6-OHDAlesioned rats. ${ }^{*} \mathrm{P}<0.001$, Mann-Whitney, comparing Vehicle + L-DOPA (dyskinetic) with doxycycline-LDOPA treated rats. Data are mean \pm SEM. DOXY: doxycycline; L-DOPA, L-3, 4-dihydroxyphenylalanine; 6-OHDA, 6-hydroxydopamine; VEH: vehicle; ALO AIMs: global AIMs score including all AIM categories (axial, forelimb, and orofacial movements).

Figure 2. NG2 distribution in striatum. A:Experimental design line. The striata was divided in dorsomedial (DM), dorsolateral (DL), ventromedial (VM) and ventrolateral (VL) for quantification analysis. B-E : Representative sections showing NG2-glia immunostaining in the DL striatum from rats without lesion (B- No lesion); lesioned with 6-OHDA toxin (C- 6OHDA); lesioned with 6-OHDA+Vehicle+ LDOPA treatment (D- 6OHDA+Vehicle +L-DOPA); dyskinetic rats treated with doxycycline $(\mathbf{E}-6 \mathrm{OHDA}+\mathrm{L}-$ DOPA+DOXY). Bar represents 20um. F-I : Quantification of NG2-glia by optic density (One-Way ANOVA) in the DM, DL, VM, VL striatum of No lesion, 6OHDA, 6OHDA+DOXY, 6OHDA+Vehicle+L-DOPA and 
6OHDA+DOXY+L-DOPA experimental groups. J-M : Spearman's rank correlation coefficient of NG2-glia with global AIMs scores axial, forelimb, and orofacial dyskinesia scores in the DM, DL, VM, VL parts of striatum. N : Relative NG2-glia protein expression level (One-Way ANOVA) in DL striatum measured by Western blot (NG2: 26kDa; $\beta$ actin: $54 \mathrm{kDa}$ ) in No lesion, 6OHDA, 6OHDA+DOXY, 6OHDA+Vehicle+LDOPA and $6 \mathrm{OHDA}+\mathrm{DOXY}+\mathrm{L}-\mathrm{DOPA}$ experimental groups of rats. ${ }^{*} \mathrm{P}<0.05,6 \mathrm{OHDA}+\mathrm{LDOPA}$ vs. all other groups. ${ }^{* *} \mathrm{P}<0.001,6 \mathrm{OHDA}+\mathrm{DOXY}+\mathrm{L}-\mathrm{DOPA}$ vs. $6 \mathrm{OHDA}+$ Vehicle$+\mathrm{LOPA} . \# \mathrm{P}<0.05$, vs. No lesion. Data are mean \pm SEM. DOXY: doxycycline; L-DOPA, L-3, 4-dihydroxyphenylalanine; 6-OHDA, 6-hydroxydopamine. $\mathrm{DL}=$ dorsolateral, $\mathrm{DM}=$ dorsomedial, $\mathrm{VM}=$ ventromedial; $\mathrm{VL}=$ ventrolateral.

Figure 3. NG2-glia undergoes morphological changes in the striata of rats presenting AIMs. A: Ramification analysis figures; $\mathbf{A}^{\prime}$ : Representative image of a NG2-glia cell (green) used for the morphological analysis. Bar represents 5um. A ":Illustration of skeletonized cell; used for analysis to get information of the cell morphology complexity. A ' ' Illustration of Sholl analysis used to quantify process branching. For quantification, the ramification index (RI) was calculated using the following formula: $\mathrm{RI}=$ convex area/cell area (Heppner et al., 1998), where convex area is the area of a polygonal object that is defined by the cells' most prominent projections and cell area is the area from soma. B-E: Data from the ramification index of rats with L-DOPA-induced dyskinesia (6OHDA+Vehicle+L-DOPA) and treated with Doxycycline $(6 \mathrm{OHDA}+\mathrm{DOXY}+\mathrm{L}-\mathrm{DOPA})$ in dorsomedial $(\mathrm{DM})$, dorsolateral $(\mathrm{DL})$, ventromedial $(\mathrm{VM})$ and ventrolateral (VL) striata areas. F: Morphometry analysis data summary for DM, DL, VM and VL striatal areas including number of branches, number of junctions, average branch length and average soma area analyzed. ${ }^{*} \mathrm{P}<0.001$ (One-Way ANOVA), vs. 6OHDA+Vehicle+LOPA. ${ }^{*} \mathrm{P}<0.001$, vs. 6OHDA. Data are mean \pm SEM. DOXY: doxycycline; L-DOPA, L-3, 4-dihydroxyphenylalanine; 6-OHDA, 6-hydroxydopamine. DL= dorsolateral, $\mathrm{DM}=$ dorsomedial, $\mathrm{VM}=$ ventromedial; $\mathrm{VL}=$ ventrolateral.

Figure 4. NG2-glia and GFAP double labeling in the striatum. A-D : Graphs show the percentage of NG2-glia co-labeling with GFAP in the total of NG2-glia in dorsomedial (DM), dorsolateral (DL), ventromedial (VM) and ventrolateral (VL) striatum.E-I : Representative sections showing NG2-glia (green), GFAP (red) staining and a merged image in DL striatum from No lesion, 6OHDA, 6OHDA+DOXY, $6 \mathrm{OHDA}+$ Vehicle+L-DOPA and 6OHDA+DOXY+L-DOPA experimental groups of rats. Bar represents 20um. Data are mean \pm SEM. DOXY: doxycycline; L-DOPA, L-3, 4-dihydroxyphenylalanine; 6-OHDA, 6-hydroxydopamine.

Figure 5. NG2-glia and OX-42 double labeling in the striatum. A-D : Graphs show the percentage of NG2-glia co-labeling with OX-42 in the total of NG2-glia in dorsomedial (DM), dorsolateral $(\mathrm{DL})$, ventromedial $(\mathrm{VM})$ and ventrolateral (VL) striatum.E-I : Representative sections showing NG2-glia (green), OX-42 (red) staining and a merged image in DL striatum from No lesion, 6OHDA, 6OHDA+DOXY, $6 \mathrm{OHDA}+$ Vehicle+L-DOPA and 6OHDA+DOXY + L-DOPA groups of rats. Bar represents 20um. Data are mean \pm SEM. DOXY: doxycycline; L-DOPA, L-3, 4-dihydroxyphenylalanine; 6-OHDA, 6-hydroxydopamine. 

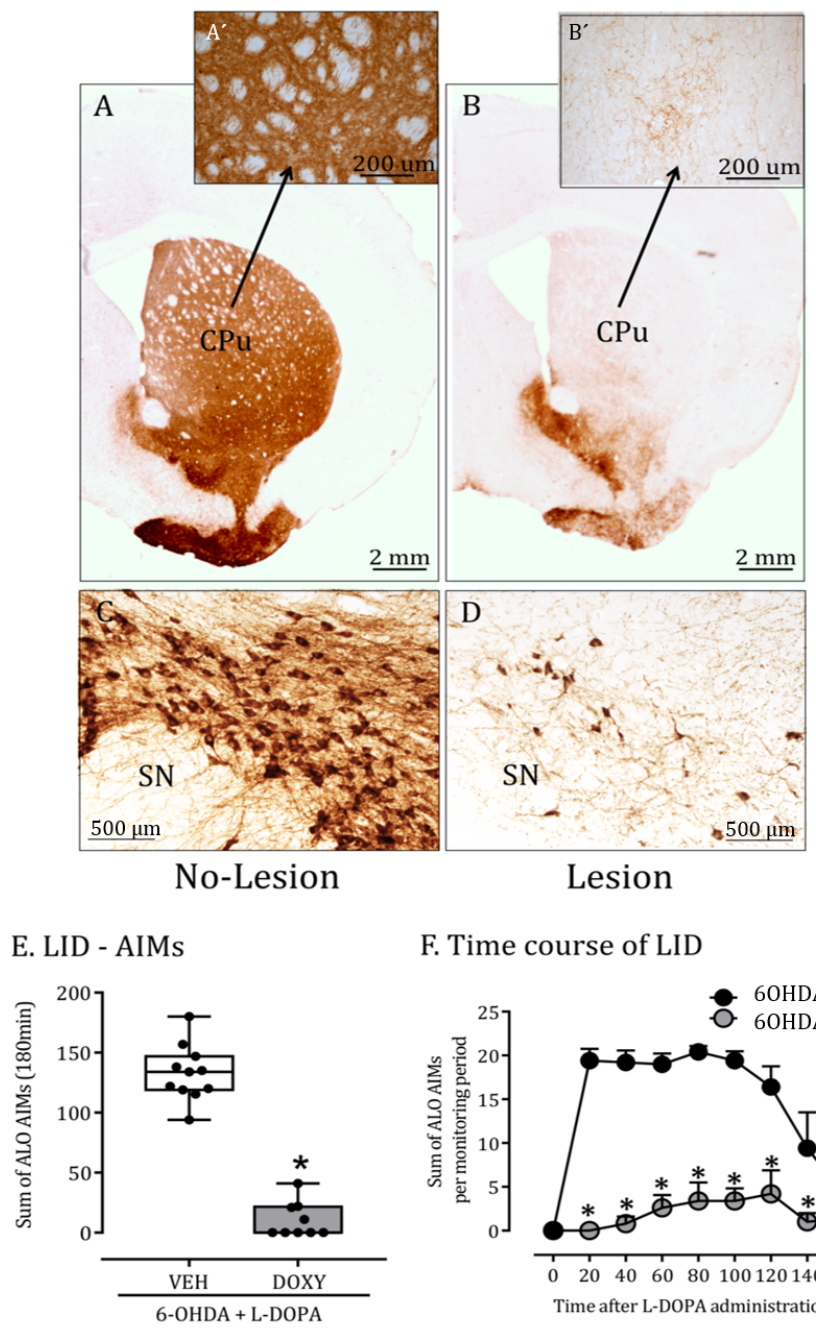

F. Time course of LID

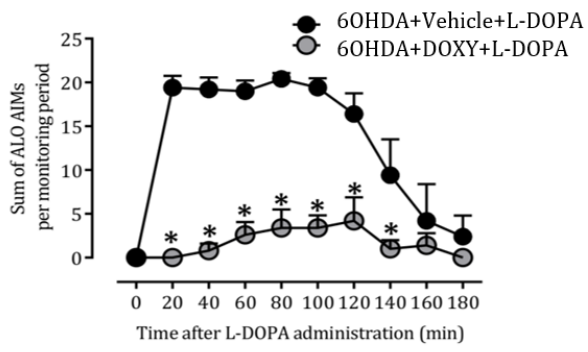

Figure 1: This is a caption 
A - Experimental Design

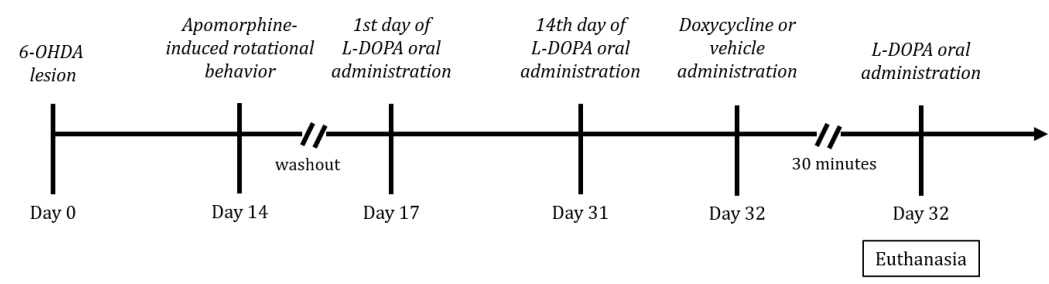

B - No Lesion

C - 6OHDA

D - 60HDA+Vehicle+

$\mathrm{E}-6 \mathrm{OHDA}+\mathrm{DOXY}+$
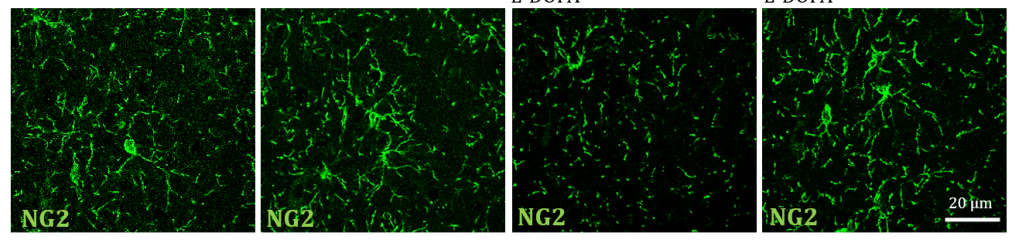

F - NG2-ir in DM striatum

G-NG2-ir in DL striatum $\quad \mathrm{H}-\mathrm{NG} 2$-ir in VM striatum

- NG2-ir in VL striatum
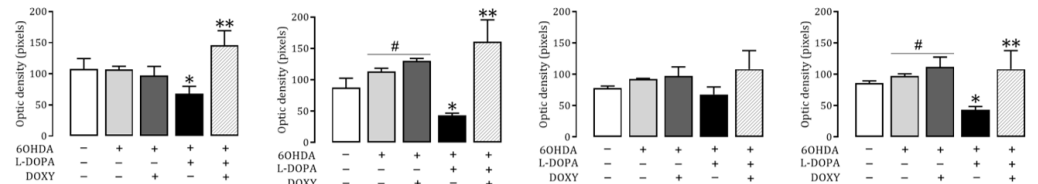

J - NG2 x Dyskinesia DM striatum

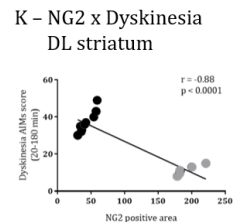

L - NG2 x Dyskinesia VM striatum

M - NG2 x Dyskinesia

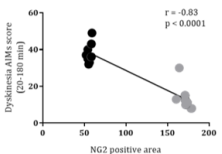

$60 H D A+V e h i c l e+L-D O P A$

6OHDA+DOXY+L-DOPA
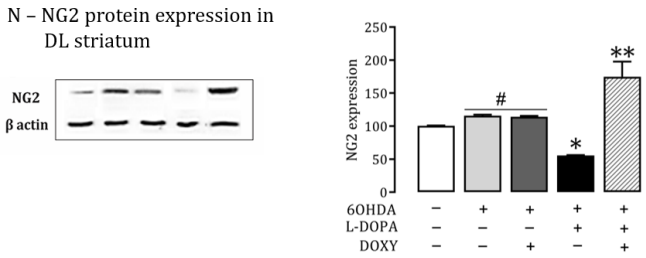

Figure 2: This is a caption 
A. Ramification analysis
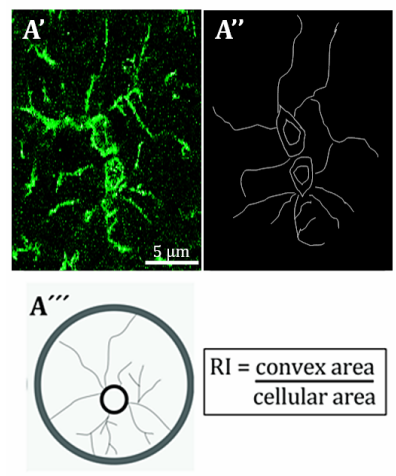

B. RI - DM

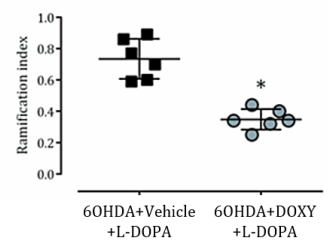

D. RI - VM

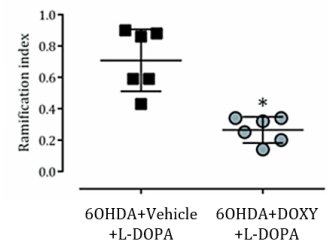

C. RI - DL

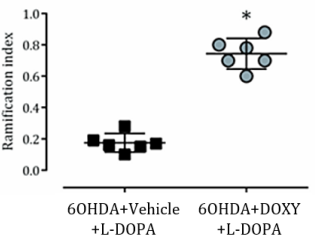

E. RI - VL

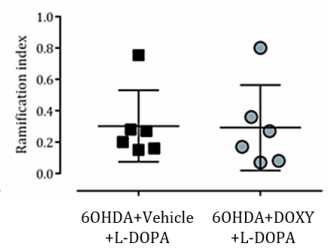

F - Skeletal analysis summary

\begin{tabular}{|c|c|c|c|c|c|}
\hline \multicolumn{6}{|l|}{ DM Striatum } \\
\hline Experimental Groups & No Lesion & 6OHDA & $60 \mathrm{HDA}+\mathrm{DOXY}$ & $\begin{array}{l}\text { 60HDA+Vehicle } \\
\text { +L-DOPA }\end{array}$ & $\begin{array}{l}\text { 6OHDA+DOXY } \\
\text { +L-DOPA }\end{array}$ \\
\hline Number of branches per cell & $5.3 \pm 0.8$ & $6.7 \pm 0.9$ & $5.8 \pm 0.4$ & $11.3 \pm 1.1^{\#}$ & $8.1 \pm 0.6^{* \#}$ \\
\hline Number of junctions per cell & $4.2 \pm 0.3$ & $3.8 \pm 0.6$ & $4.1 \pm 0.7$ & $5.6 \pm 0.9^{\#}$ & $5.1 \pm 0.8$ \\
\hline Average branch lenght $(\mu \mathrm{m})$ & $6.3 \pm 0.8^{\#}$ & $8.2 \pm 0.9$ & $7.5 \pm 1.2$ & $13.8 \pm 1.2^{\#}$ & $9.2 \pm 1.3^{* \#}$ \\
\hline Average of Soma area $\left(\mu \mathrm{m}^{2}\right)$ & $1.3 \pm 0.4$ & $2.2 \pm 0.9$ & $1.8 \pm 0.5$ & $4.1 \pm 0.8^{\#}$ & $3.8 \pm 0.2^{* \#}$ \\
\hline \multicolumn{6}{|l|}{ DL Striatum } \\
\hline Experimental Groups & No Lesion & 6OHDA & 6OHDA+DOXY & $\begin{array}{l}\text { 60HDA+Vehicle } \\
\text { +L-DOPA }\end{array}$ & $\begin{array}{l}\text { 6OHDA+DOXY } \\
\text { +L-DOPA }\end{array}$ \\
\hline Number of branches per cell & $4.5 \pm 1.1$ & $5.8 \pm 1.2$ & $5.1 \pm 0.9$ & $4.2 \pm 0.2^{\#}$ & $12.2 \pm 3.1^{* \#}$ \\
\hline Number of junctions per cell & $1.8 \pm 0.2$ & $2.8 \pm 1.1$ & $2.4 \pm 0.7$ & $3.8 \pm 0.2$ & $6.8 \pm 1.7^{* \#}$ \\
\hline Average branch lenght $(\mu \mathrm{m})$ & $5.5 \pm 1.3$ & $7.1 \pm 0.8$ & $9.5 \pm 1.4^{\#}$ & $4.2 \pm 1.2^{\#}$ & $13.5 \pm 1.1^{* \#}$ \\
\hline Average of Soma area $\left(\mu \mathrm{m}^{2}\right)$ & $1.2 \pm 0.3^{\#}$ & $2.2 \pm 0.2$ & $1.9 \pm 0.5$ & $2.3 \pm 1.3$ & $5.2 \pm 0.9^{* \#}$ \\
\hline \multicolumn{6}{|l|}{ VM Striatum } \\
\hline Experimental Groups & No Lesion & 6OHDA & $60 \mathrm{HDA}+\mathrm{DOXY}$ & $\begin{array}{l}\text { 60HDA+Vehicle } \\
\text { +L-DOPA }\end{array}$ & $\begin{array}{l}\text { 6OHDA+DOXY } \\
\text { +L-DOPA }\end{array}$ \\
\hline Number of branches per cell & $4.3 \pm 0.6$ & $5.7 \pm 0.6$ & $5.6 \pm 0.4$ & $9.3 \pm 0.5^{\#}$ & $7.1 \pm 0.4^{* \#}$ \\
\hline Number of junctions per cell & $3.2 \pm 0.4$ & $2.8 \pm 0.4$ & $2.1 \pm 0.6$ & $4.8 \pm 0.7^{\#}$ & $3.1 \pm 1.2$ \\
\hline Average branch lenght $(\mu \mathrm{m})$ & $5.3 \pm 0.9$ & $6.2 \pm 0.7$ & $6.5 \pm 1.3$ & $12.6 \pm 1.1^{\#}$ & $8.9 \pm 1.1^{* \#}$ \\
\hline Average of Soma area $\left(\mu \mathrm{m}^{2}\right)$ & $1.1 \pm 0.7$ & $1.9 \pm 0.8$ & $1.6 \pm 0.3$ & $3.1 \pm 0.4$ & $3.6 \pm 0.8^{\#}$ \\
\hline \multicolumn{6}{|l|}{ VL Striatum } \\
\hline Experimental Groups & No Lesion & 6OHDA & 6OHDA+DOXY & $\begin{array}{l}\text { 60HDA+Vehicle } \\
\text { +L-DOPA }\end{array}$ & $\begin{array}{l}\text { 6OHDA+DOXY } \\
\text { +L-DOPA }\end{array}$ \\
\hline Number of branches per cell & $4.3 \pm 0.9$ & $4.7 \pm 0.6$ & $4.6 \pm 0.3$ & $4.3 \pm 0.5$ & $4.1 \pm 0.6$ \\
\hline Number of junctions per cell & $2.2 \pm 0.8$ & $2.3 \pm 0.2$ & $2.1 \pm 0.4$ & $2.8 \pm 0.6$ & $2.1 \pm 0.2$ \\
\hline Average branch lenght $(\mu \mathrm{m})$ & $5.3 \pm 0.9$ & $6.2 \pm 0.7$ & $6.5 \pm 1.3$ & $6.6 \pm 1.1$ & $6.9 \pm 1.1$ \\
\hline Average of Soma area $\left(\mu \mathrm{m}^{2}\right)$ & $1.1 \pm 0.7$ & $1.9 \pm 0.8$ & $1.6 \pm 0.3$ & $2.1 \pm 0.4$ & $1.6 \pm 0.8$ \\
\hline
\end{tabular}

Figure 3: This is a caption 


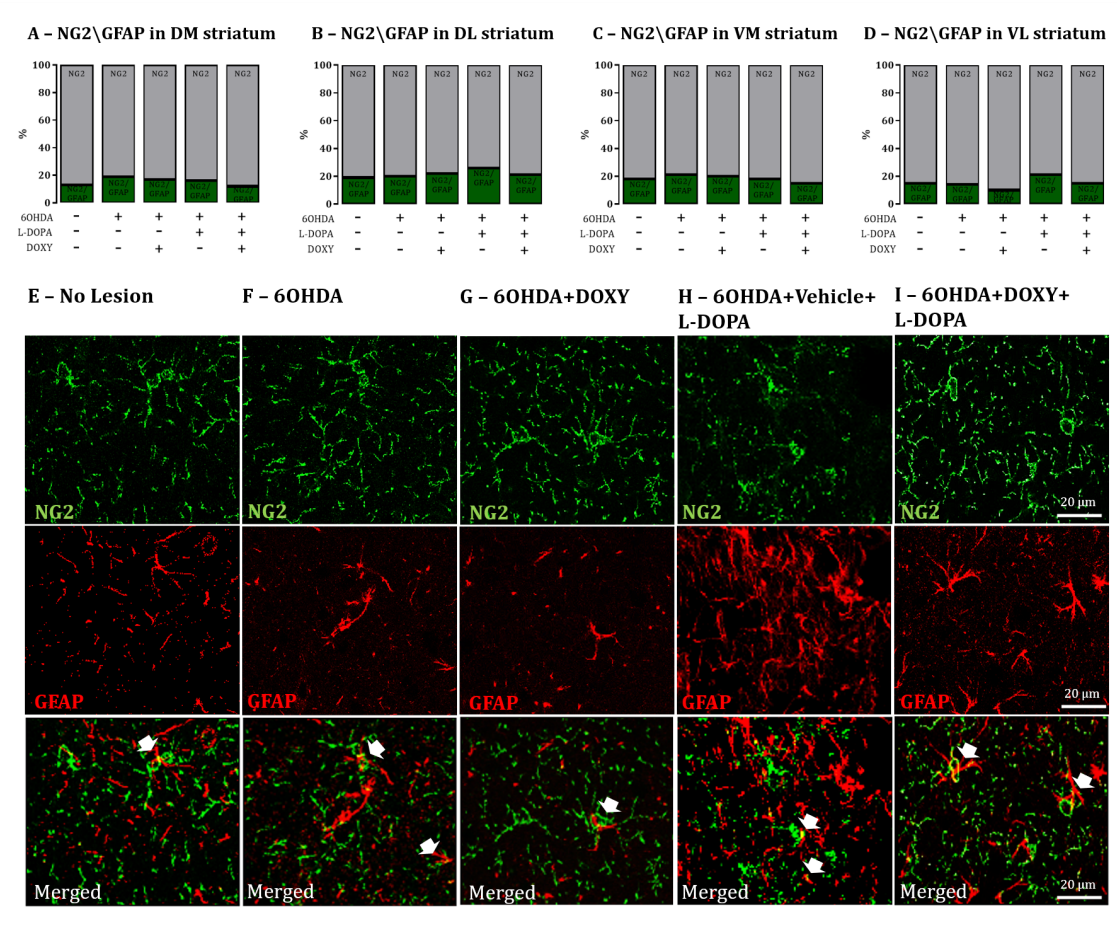

Figure 4: This is a caption

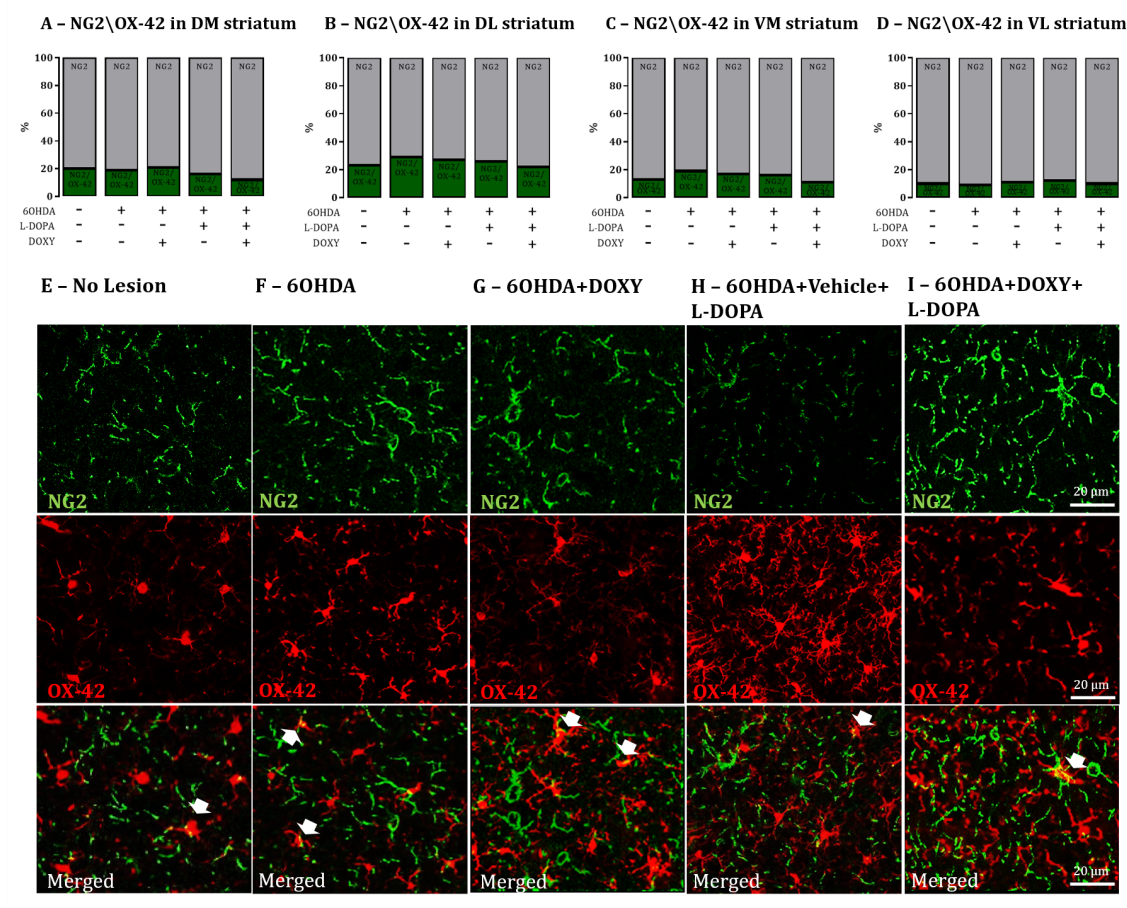

Figure 5: This is a caption 